\title{
An effective multi-channel fault diagnosis approach for rotating machinery based on multi- variate generalized refined composite multi-scale sample entropy
}

\section{Zhenya Wang}

Fuzhou University

Hui Chen

Fuzhou University

Ligang Yao ( $\nabla$ ylgyao@fzu.edu.cn )

Fuzhou University https://orcid.org/0000-0002-7183-7794

\section{Xu Chen}

Anhui University of Technology

Xiaoli Qi

Anhui University of Technology

Jun Zhang

Fuzhou University

\section{Original Research}

Keywords: Fault diagnosis, Rotating machine, Multi-scale entropy, Isometric mapping, Support vector machine

Posted Date: February 1st, 2021

DOI: https://doi.org/10.21203/rs.3.rs-179198/v1

License: (c) (1) This work is licensed under a Creative Commons Attribution 4.0 International License. Read Full License 


\title{
An effective multi-channel fault diagnosis approach for rotating machinery based on multi-variate generalized refined composite multi-scale sample entropy
}

\author{
Zhenya Wang $\cdot$ Hui Chen $\cdot$ Ligang Yao $\cdot$ Xu Chen $\cdot$ Xiaoli Qi $\cdot$ Jun Zhang
}

Received: data / Accepted: data

\begin{abstract}
Fault diagnosis of critical rotating machinery components is necessary to ensure safe operation. However, the commonly used fault diagnosis methods for rotating machinery are generally based on the single-channel signal processing method, which is not suitable for processing multi-channel signals. Thus, to extract features and carry out the intelligent diagnosis of multi-channel signals, a novel method for rotating machinery fault diagnosis is proposed. Firstly, a novel non-linear dynamics technique, named the multi-variate generalized refined composite multi-scale sample entropy, is presented and applied to extract fusion entropy features of multi-channel signals. Secondly, a practical manifold learning known as supervised isometric mapping is introduced to map the high-dimensional fusion entropy features in a low-dimensional space. In a third step, the Harris hawks optimization-based support vector machine is utilized to achieve intelligent fault recognition. Finally, aiming to verify the effectiveness of the proposed method and present its advantages, it was applied to analyze the rotating machinery system bearing and gear data. The experimental results have shown that the method at hand can accurately identify various bearing and gear faults. Furthermore, in addition to being suitable for multi-channel signal fault diagnosis, it displayed higher recognition accuracy when compared with other multi-channel or single-channel methods.
\end{abstract}

Keywords Fault diagnosis $\cdot$ Rotating machine $\cdot$ Multi-scale entropy $\cdot$ Isometric mapping $\cdot$ Support vector machine

Z. Wang $\cdot$ H. Chen $\cdot$ L. Yao $(\bowtie) \cdot$ J. Zhang $(\bowtie)$

School of Mechanical Engineering and Automation, Fuzhou University, Fuzhou 350108, PR China

Z. Wang

e-mail:wzyaxj@163.com

L. Yao

e-mail: ylgyao@fzu.edu.cn

J. Zhang

e-mail: zhang_jun@fzu.edu.cn

X. Chen · X. Qi

School of Mechanical Engineering, Anhui University of Technology, Maanshan 243032, PR China 


\section{Introduction}

Rotating machinery has been widely employed in various industrial fields, including petroleum, aerospace, metallurgy, energy, and chemistry. As critical rotating machinery components, bearings and gears have a significant role when it comes to support, power conversion, and power transmission. However, due to the complex operating conditions, their performance gradually degrades in the long-term operation and faults occur. Such behavior affects the rotating machinery performance, reliability, and service life, often causing unavoidable economic losses. Thus, it is necessary to implement an effective intelligent fault diagnosis method for both bearings and gears [1-3].

\section{1 Motivation}

During the previous two decades, intelligent fault diagnosis methods based on vibration signals were gradually developed and subsequently applied in rotating machinery. Such methods are commonly used as they were proven to be effective [4]. They mostly contain two steps: (1) the signal processing algorithms are firstly utilized to extract fault features; (2) various pattern recognition algorithms are applied to diagnose the faults based on the extracted features [5]. Commonly used intelligent fault diagnosis methods are often based on single-channel signals [6-10].

However, for rotating machinery, a single information source often contains limited fault information, meaning that it may lead to ambiguous or even wrong diagnosis. Additionally, with the continuous improvement of rotating machinery safety requirements, this type of technology cannot meet the growing demand for data and information mining [11]. Thus, the fusion data processing methods based on multi-channel signals have attracted the attention of scholars aiming to detect and diagnose the situation in detail. Such multi-channel signal processing methods are slowly becoming inevitable in the development of intelligent fault diagnosis [12, 13]. For these technologies, signals obtained by the multiple sensors are processed as a whole, which enables them to fully extract the associated information, improve the information utilization rate, and finally, obtain more accurate and reliable diagnosis results.

Moreover, the fusion features obtained using the multi-channel rotating machinery signals are regularly high-dimensional, non-linear, and are inevitably mixed with redundancy. Such properties increase the recognition time and reduce the diagnostic accuracy of subsequent classifiers [14]. When it comes to selecting low-dimensional and sensitive feature sets from these massive and non-linear fusion features, it is imperative to consider intelligent fault diagnosis methods.

\section{2 Entropy-based feature extraction approach}

The rotating machinery vibration signals are usually non-linear and non-stationary, significantly increasing the fault feature extraction complexity [14]. Fortunately, with the continuous development of non-linear theory, entropy-based feature extraction algorithms can overcome the problem, as mentioned earlier. As such, they are widely applied for the feature extraction of various mechanical signals. Said technology mainly includes approximate entropy, sample entropy, permutation entropy, fuzzy entropy, and dynamic symbolic entropy [15-19].

Sample entropy is a frequently used method, which can effectively detect the minuscule time series changes. When the rotating machinery faults occur, the corresponding non-linear dynamics complexity changes accordingly, requiring the application of sample entropy-based feature extraction algorithms [20, 21-23]. For example, Wang combined the sample entropy method with ensemble empirical mode decomposition to identify the rolling bearing faults [21]. Gao et al. combined multi-scale sample entropy with local mean decomposition to effectively extract the wind turbine fault features [22]. Furthermore, Dai et al. proposed a composite multi-scale sample entropy algorithm developed by combining the composite coarse-grained structure and the multi-scale sample entropy. The algorithm 
was proven to extract the bearing features effectively [23]. Recently, Wang et al. proposed the generalized refined composite multi-scale sample entropy algorithm, verifying its advantages by using bearing data [20].

The above-mentioned sample entropy-based algorithms performed very well in rotating machinery fault diagnosis; however, all the algorithms belong to the group of single-channel analysis methods. Since such methods are not suitable for processing multi-channel signals, it is vital to explore the state-of-the-art multi-channel signal processing methods. Lu and Wang proposed the multi-variate multi-scale sample entropy (MMSE) algorithm based on multi-scale sample entropy [24]. The algorithm was used to analyze any number of channel signals, providing reliable complexity analysis results. Nevertheless, when using MMSE to extract the fusion features of multi-channel rotating machinery signals, two shortcomings remained. (1) The new multi-variate coarse-grained structure (MCS) of MMSE only considers the single MCS under the scale factor $s$, ignoring other equally important MCSs. (2) MMSE obtains a new MCS by sliding a window and averaging all the data within, which can cause the amplitude information of multi-channel signals to be ignored [25-26].

However, studies were carried out, aiming to improve the MMSE. For example, to solve the first shortcoming, Humeau-Heurtier proposed the multi-variate refined composite multi-scale sample entropy (MRCMSE) algorithm. The algorithm considers multiple MCSs (i.e., multi-variate composite coarse-grained structures) under $s$. Furthermore, the refined operation is used to avoid invalid entropy values [25]. Additionally, regarding the second deficiency, Yin et al. introduced the multi-variate generalized multi-scale sample entropy (MGMSE). The original average calculation method applied in MMSE was replaced by variance operation in MCS construction of MMSE. The authors demonstrated the MGMSE effectiveness by distinguishing traffic detectors of various complexities [26].

Unfortunately, both MRCMSE and MGMSE only solve one MMSE shortcoming. Thus, a novel MGRCMSE algorithm is proposed in this paper, aiming to overcome both defects by combining MRCMSE and MGMSE. To prove its effectiveness, the algorithm was applied to extract the fusion feature of multi-channel vibration signals.

\section{3 The proposed multi-channel fault diagnosis method}

In this study, the authors have proposed an intelligent fault diagnosis method based on the fusion entropy-manifold features and optimized support vector machine. The proposed method enables the effective mining of the fusion features from multi-channel rotating machinery signals, increasing the fault diagnosis accuracy, which is the main contribution of this paper. The method is divided into three phases:

- The first phase - a novel non-linear algorithm, named multi-variate generalized refined composite multi-scale sample entropy (MGRCMSE) was developed to enable mining the fusion features from multi-channel signals.

- The second phase - includes utilizing the supervised isometric mapping (SIM) [20] algorithm to reduce the fusion entropy feature dimensionality, enabling the authors to obtain the low-dimensional and sensitive fusion entropy-manifold feature sets.

- The third phase - is focused on fault identification and diagnosis; the Harris hawks optimization-based support vector machine (HHO-SVM) [27] is applied to both identify and diagnose the faults.

The effectiveness of the proposed method is verified by analyzing the fault diagnosis experiments of bearings and gears mounted within the rotating machinery system. Moreover, compared to other single-channel or multi-channel feature extraction methods, the proposed feature extraction method extracted features from the original signals more effectively and with a higher fault diagnosis ability.

\section{4 Contributions and structure of this paper}

The main contributions of this paper can be stated as follows: 
- A novel non-linear dynamics analysis algorithm (MGRCMSE) is proposed.

- The proposed algorithm is used to carry out the multi-channel fault diagnosis of the rotating machinery.

- The proposed algorithm is combined with the SIM for sensitive feature extraction.

- The simulation and fault diagnosis experiments verified the advantages of the proposed method.

Finally, the paper is structured as follows: Section 2 details the mathematical background behind the MGRCMSE, while the novel intelligent multi-channel fault diagnosis method for rotating machinery was presented in Section 3. The bearings and gears were used as test specimens in the experimental verification (Section 4). Finally, conclusions are given in Section 5.

\section{Proposed multi-variate generalized refined composite multi-scale sample entropy approach}

The MMSE and MGMSE algorithms are introduced in this section. Additionally, based on these two algorithms the MGRCMSE is proposed after implementing the multi-variate generalized composite coarse-grained structure and refined operation. The flowchart of these three algorithms allowing the easier comparison is shown in Fig. 1.

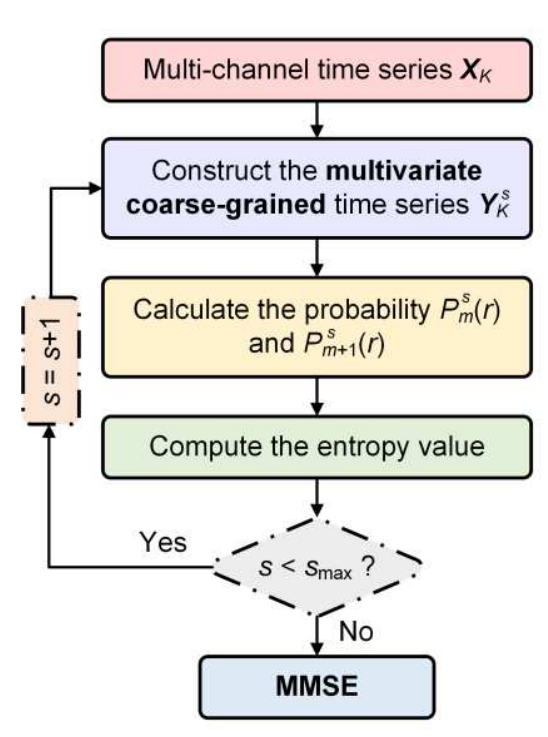

(a)

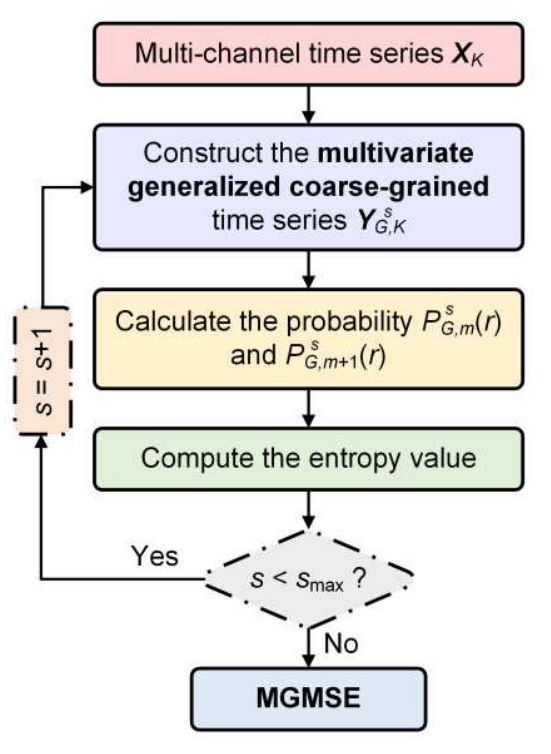

(b)

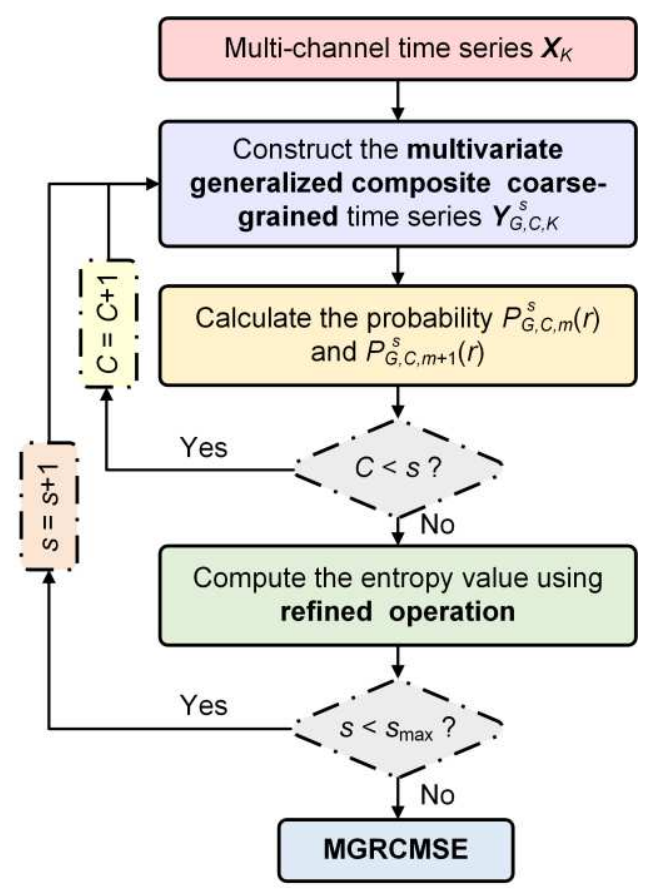

(c)

Fig. 1 Flowchart of three multi-channel feature extraction algorithms: (a) MMSE, (b) MGMSE, and (c) MGRCMSE

\section{1 Multi-variate multi-scale sample entropy}

For a multi-channel time series $\boldsymbol{X}_{K}=\left\{x_{K, i}\right\}_{i=1}^{N}, K=1,2, \ldots, p$ is the number of channels, and $N$ is the number of sample points of each channel time series. The specific MMSE steps are as follows:

(1) The MCSs $\boldsymbol{Y}_{K}^{s}=\left\{y_{K, j}^{s}\right\}_{j=1}^{N s}$ of multi-channel signals at $s$ are constructed using Eq. (1), where $N s=[N / s]$.

$$
y_{K, j}^{s}=\frac{1}{s} \sum_{i=(j-1)}^{j s} x_{K, i}
$$

(2) According to the time delay embedding theory, the corresponding delay vector $\boldsymbol{Y}_{m}^{s}(j)$ is found for each $\boldsymbol{Y}_{K}^{s}$.

$$
\boldsymbol{Y}_{m}^{s}(j)=\left[y_{1, j}^{s}, y_{1, j+\tau_{1}}^{s}, \ldots, y_{1, j+\left(m_{1}-1\right) \tau_{1}}^{s}, y_{2, j}^{s}, y_{2, j+\tau_{2}}^{s}, \ldots, y_{2, j+\left(m_{2}-1\right) \tau_{2}}^{s}, \ldots, y_{p, j}^{s}, y_{p, j+\tau_{p}}^{s}, \ldots, y_{p, j+\left(m_{p}-1\right) \tau_{p}}^{s}\right]
$$

where $\boldsymbol{M}=\left[m_{1}, m_{2}, \ldots, m_{p}\right]$ is the embedding vector, $\boldsymbol{\tau}=\left[\tau_{1}, \tau_{2}, \ldots, \tau_{p}\right]$ is the delay vector, $j=1,2, \ldots, N s-L$, and $L=\max (\boldsymbol{M}) \times \max (\boldsymbol{\tau})$. 
(3) The maximum norm value of any two delay vectors $\boldsymbol{Y}_{m}^{s}(j)$ and $\boldsymbol{Y}_{m}^{s}(h)$ is calculated as the distance between them.

$$
d\left[\boldsymbol{Y}_{m}^{s}(j), \boldsymbol{Y}_{m}^{s}(h)\right]=\max _{l=1, \ldots, m}\left\{\left|y^{s}(j+l-1)-y^{s}(h+l-1)\right|\right\}
$$

If $d\left[\boldsymbol{Y}_{m}^{s}(j), \boldsymbol{Y}_{m}^{s}(h)\right] \leq r, j \neq h$, these two vectors are similar, with $r$ being a similarity tolerance.

(4) The variables $B_{m, j}^{s}=d\left[\boldsymbol{Y}_{m}^{s}(j), \boldsymbol{Y}_{m}^{s}(h)\right] \leq r$ and $P_{m, j}^{s}(r)=B_{m, j}^{s} /(N s-L-1)$ are defined. Then, the similarity probability of any two delay vectors at the embedding dimension $m$ can be calculated using the expression below:

$$
P_{m}^{s}(r)=\frac{1}{N s-L} \sum_{j=1}^{N s-L} P_{m, j}^{s}(r)
$$

(5) The steps (1) - (4) are repeated to calculate the similarity probability of any two delay vectors at $m+1$ dimension. To ensure that the whole system is transformed into $m+1$ dimension, the embedding size of other channels remains the same. Next, $B_{m+1, j}^{s}=d\left[\boldsymbol{Y}_{m+1}^{s}(j), \boldsymbol{Y}_{m+1}^{s}(h)\right] \leq r$ and $P_{m+1, j}^{s}(r)=B_{m+1, j}^{s} /[p(N s-L)-1]$ are defined, followed by the calculation of the similarity probability of any two delay vectors at the embedding dimension $m+1$ using Eq. (5):

$$
P_{m+1}^{s}(r)=\frac{1}{p(N s-L)} \sum_{j=1}^{p(N s-L)} P_{m+1, j}^{s}(r)
$$

(6) The final MMSE values can be computed by:

$$
\operatorname{MMSE}\left(\boldsymbol{X}_{K}, s, m, r, N\right)=-\ln \left[\frac{P_{m+1}^{s}(r)}{P_{m}^{s}(r)}\right]
$$

\section{2 Multi-variate generalized multi-scale sample entropy}

In the MGMSE algorithm, the variance calculation is used to replace the average calculation in MMSE, retaining the time series amplitude information. For the multi-channel time series $\boldsymbol{X}_{K}=\left\{x_{K, i}\right\}_{i=1}^{N}$, the main MGMSE steps can be summarized as follows:

(1) The multi-variate generalized coarse-grained structures $\boldsymbol{Y}_{G, K}^{s}=\left\{y_{G, K, j}^{s}\right\}_{j=1}^{N s}$ of multi-channel signals at $s$ are constructed:

$$
\begin{gathered}
y_{G, K, j}^{s}=\frac{1}{s} \sum_{i=(j-1) s+1}^{j s}\left(x_{K, i}-\overline{x_{K, i}}\right)^{2} \\
\overline{x_{K, i}}=\frac{1}{s} \sum_{i=(j-1) s+1}^{j s} x_{K, i}
\end{gathered}
$$

(2) Similarly, for each $\boldsymbol{Y}_{G, K}^{s}$, the similarity probabilities $P_{G, m}^{s}(r)$ and $P_{G, m+1}^{s}(r)$ can be calculated by applying the MMSE steps (2) to (5).

(3) Lastly, the MGMSE output values can be computed as:

$$
\operatorname{MGMSE}\left(\boldsymbol{X}_{K}, s, m, r, N\right)=-\ln \left[\frac{P_{G, m+1}^{s}(r)}{P_{G, m}^{s}(r)}\right]
$$

\section{3 Multi-variate generalized refined composite multi-scale sample entropy}

Based on MGMSE, in this paper, we propose the MGRCMSE algorithm, which was developed combining the multi-variate composite coarse-grained structure and refined entropy operation. For $\boldsymbol{X}_{K}=\left\{x_{K, i}\right\}_{i=1}^{N}$, the key MGRCMSE steps are: 
(1) The multi-variate generalized composite coarse-grained structures $\boldsymbol{Y}_{G, C, K}^{s}=\left\{y_{G, C, K, j}^{s}\right\}_{j=1}^{N s}$ of multi-channel signals at $s$ are found using Eq. (9), where $C=1,2, \ldots, s$ :

$$
\begin{gathered}
y_{G, C, K, j}^{s}=\frac{1}{s} \sum_{i=(j-1) s+C}^{j s+C-1}\left(x_{K, i}-\overline{x_{K, i}}\right)^{2} \\
\overline{x_{K, i}}=\frac{1}{s_{i=(j-1) s+C}} \sum_{K, i}^{j s+C-1} x_{1}
\end{gathered}
$$

(2) For each $\boldsymbol{Y}_{G, C, K}^{s}$, the similarity probabilities $P_{G, C, m}^{s}(r)$ and $P_{G, C, m+1}^{s}(r)$ are calculated according to the MMSE steps (2) to (5).

(3) The final MGRCMSE values can be computed by using refined entropy operation:

\section{4 Simulation analysis}

$$
\operatorname{MGRCMSE}\left(\boldsymbol{X}_{K}, s, m, r, N\right)=-\ln \left[\frac{\sum_{C=1}^{s} P_{G, C, m+1}^{s}(r)}{\sum_{C=1}^{s} P_{G, C, m}^{s}(r)}\right]
$$

The MGRCMSE parameter settings are determined by carrying out noise experiments, allowing the authors to compare its performance and effectiveness to the MMSE, MRCMSE, and MGMSE.

\section{4. 1 MGRCMSE parameter selection}

In the MGRCMSE, four parameters including the data length $N$, embedding dimension $m$, similarity tolerance $r$, and scale factor $s$ must be set manually. To study the influence these parameters have on the MGRCMSE, three-channel white noise and three-channel pink noise was utilized.

(1) Influence of the scale factor s on MGRCMSE. There is no standard for adjusting the parameter $s$; therefore, in this paper, the authors set its value to $s=25$.

(2) Influence of the data length $N$ on MGRCMSE. MGRCMSE is used to analyze three-channel noise signals with different lengths; $N=\{2000,3000,4000,5000\}$. The entropy curve results for $m=2, r=0.15 s d$ (where $s d$ is the time-series standard deviation), and $s=25$ are plotted in Fig. 2.

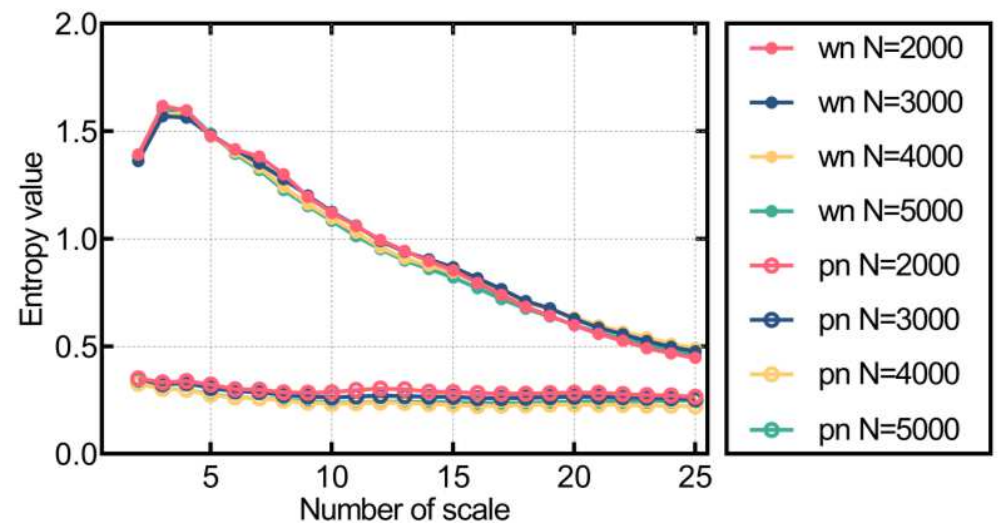

Fig. 2 Influence of $N$ on MGRCMSE

According to Fig. 2, the fusion entropy values of the three-channel white noise are higher than that those of the three-channel pink noise for the majority of $N$ values. The reason for this phenomenon is because, compared to pink noise, white noise has stronger randomness and weaker self-similarity; therefore, its entropy values are higher. Furthermore, the three-channel white noise (or three-channel pink noise) entropy curves are similar to each other regardless of $N$, meaning that it has a limited effect on the MGRCMSE. Thus, its value was set to $N=3000$.

(3) The influence of the embedding dimension $m$ on MGRCMSE. By selecting four different $m$ values as $m=\{1,2$, $3,4\}$, the MGRCMSE is used to analyze two above-mentioned noise signals. The resulting entropy curves 
obtained using $N=3000, r=0.15 s d$, and $s=25$ are shown in Fig. 3 .
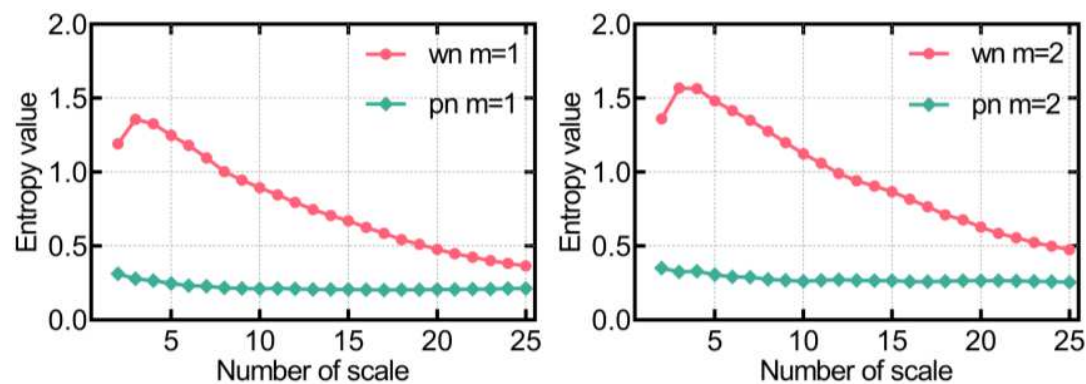

(a)

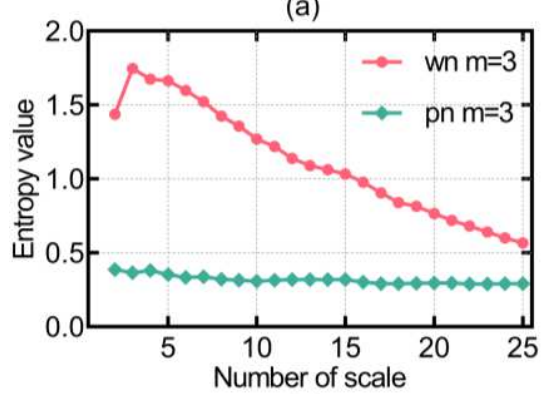

(c)

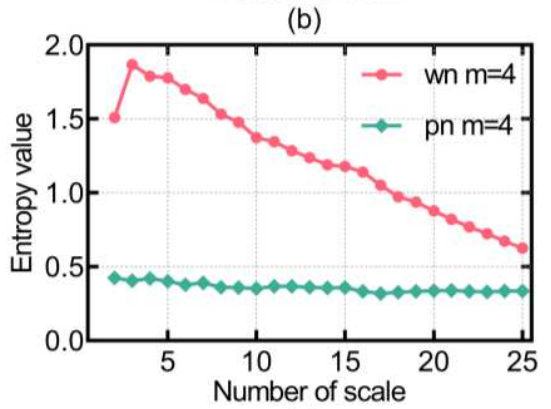

(d)

Fig. 3 Influence of $m$ on MGRCMSE: (a) $m=1$, (b) $m=2$, (c) $m=3$, and (d) $m=4$

It can be seen from Fig. 3 that, primarily, both the white and pink noise entropy values increase with the increase in $m$. However, the overall trend practically remains the same. Secondly, when $m$ is a small value (for example, $m=1$ ), the white and pink noise entropy curves have similar values on large scales. On the other hand, when $m$ is a large value (i.e., $m=3$ or 4 ), the entropy curves fluctuate noticeably. Finally, when $m$ is 2 , the MGRCMSE entropy curves of both noise signals are relatively smooth, allowing us to distinguish between them on the large scales. Therefore, the value $m=2$ was used in this paper.

(4) The influence of the similarity tolerance $r$ on MGRCMSE. Four different $r$ values $(0.1 s d, 0.15 s d, 0.2 s d$, and $0.25 s d$ ) were used to find the optimal one; the MGRCMSE is applied to analyze the above-mentioned noise signals. Fig. 4 illustrates the entropy curves for $N=3000, m=2$, and $s=25$.
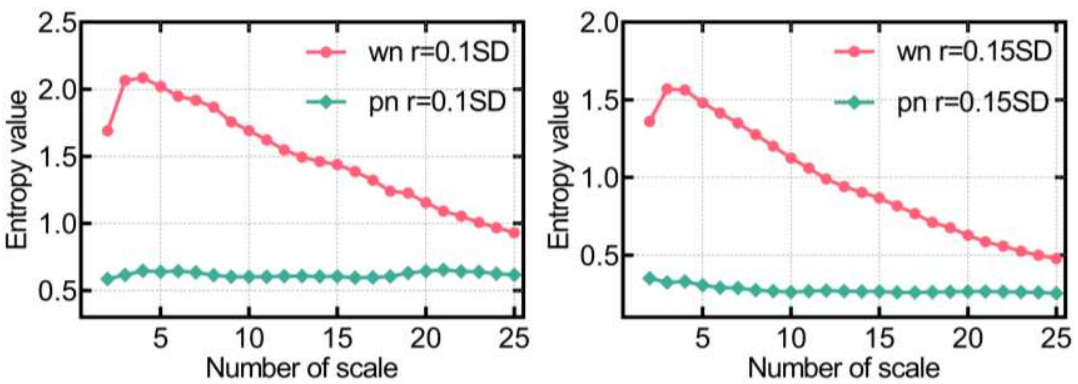

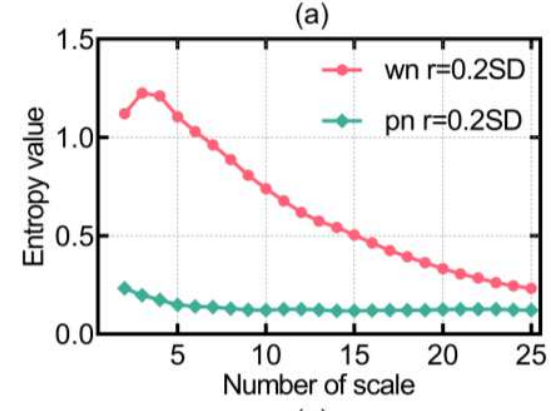

(c)

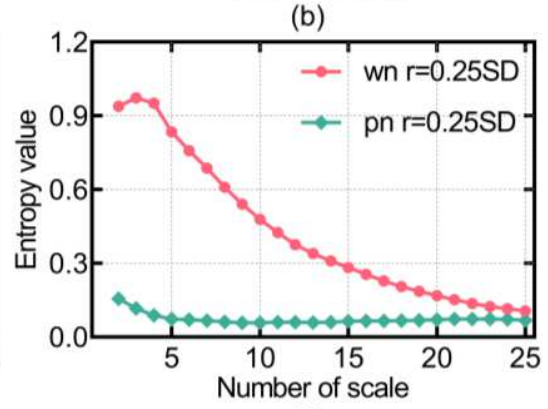

(d)

Fig. 4 Influence of $r$ on MGRCMSE: (a) $r=0.1 s d$, (b) $r=0.15 s d$, (c) $r=0.2 s d$, and (d) $r=0.25 s d$

It is evident from Fig. 4 that for the same noise signal, the MGRCMSE entropy curve decreases with the increase in $r$. This can be explained by the fact that, when $r$ value is low, the number of matching templates increases, 
increasing the entropy values. Moreover, when $r$ is a small value (e. g., $r=0.1 s d$ ), the MGRCMSE entropy curves of both noise signals have slight fluctuation. On the other hand, the number of matching templates decreases when $r$ is a large value, reducing the entropy values. When $r$ is large (e. g., $r=0.25 s d$ ), the MGRCMSE entropy curves of both noise signals are relatively close in the large scales. When $r$ is $0.15 s d$ or $0.2 s d$, the MGRCMSE entropy curves of both groups of signals are relatively smooth, and the noise signals can be distinguished on large scales. Therefore, $r$ $=0.15 s d$ was used in this paper.

To conclude, within the paper, the MGRCMSE parameters are set to $N=3000, m=2, r=0.15 s d$, and $s=25$.

\section{4. 2 Comparison of the various multi-channel signal analysis algorithms}

In this section, the MGRCMSE algorithm is compared with the MMSE, MRCMSE, and MGMSE algorithms to assess its effectiveness. Four three-channel signals (as shown in Table 1) were analyzed using each of the algorithms, and the analysis results are shown in Fig. 5. It should be noted that all the algorithm parameter values were consistent with the MGRCMSE parameters.

Table 1 Four groups of three-channel noise signals

\begin{tabular}{ll}
\hline Abbreviation & Description \\
\hline 3 wn & Three-channel white noise \\
3 pn & Three-channel pink noise \\
1 wn 2 pn & One-channel white noise and two-channel pink noise \\
2 wn 1 pn & Two-channel white noise and one-channel pink noise \\
\hline
\end{tabular}

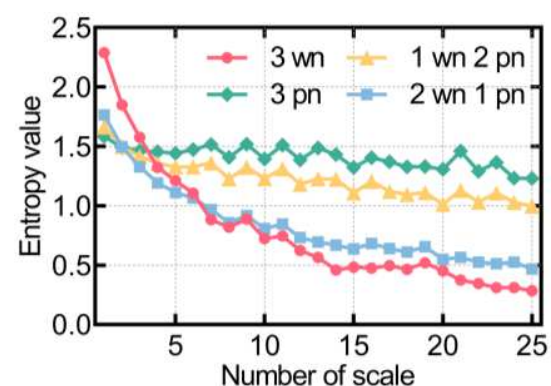

(a)

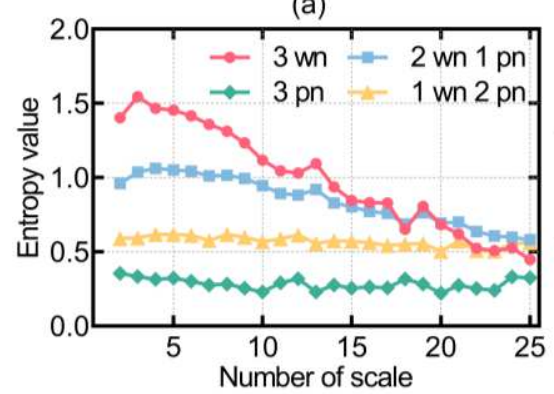

(c)

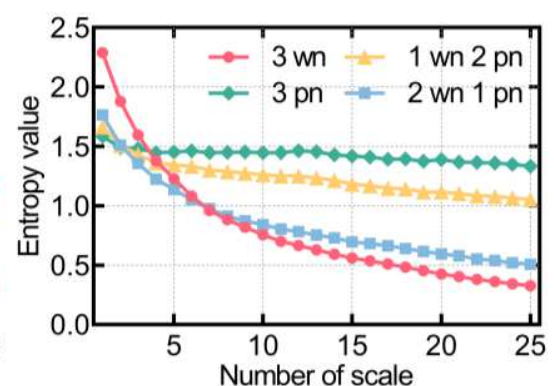

(b)

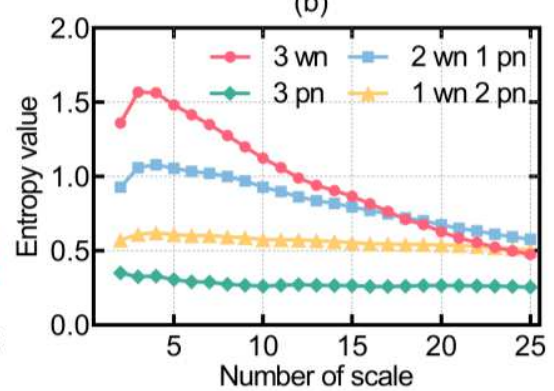

(d)

Fig. 5 Analysis results of four multi-channel signal analysis algorithms: (a) MMSE, (b) MRCMSE, (c) MGMSE, and (d) MGRCMSE

Several conclusions were drawn from Fig. 5. Firstly, the entropy curves obtained by the MGRCMSE (or MRCMSE) for each group of signals have displayed lower fluctuation compared with the MGMSE (or MMSE). This is since MGRCMSE (or MRCMSE) adopts the multi-variate refined composite coarse-grained structure, allowing it to comprehensively consider multiple sequences at $s$. At the same time, the MGMSE (or MMSE) only considers the single coarse-grained sequence, ignoring the other important MCSs information. Thus, the MGRCMSE-obtained (or MRCMSE) entropy values were more stable, further confirming the advantages of the multi-variate refined 
composite coarse-grained structure.

Next, for the MRCMSE (or MMSE), the entropy values of the four group signals are ordered as follows: $E_{3 p n}>$ $E_{1 w n 2 p n}>E_{2 w n 1 p n}>E_{3 w n}$, while in the MGRCMSE (or MGMSE), it is $E_{3 w n}>E_{2 w n 1 p n}>E_{1 w n 2 p n}>E_{3 p n}$. In practice, the signal irregularity values are ordered as $I_{3 w n}>I_{2 w n 1 p n}>I_{1 w n 2 p n}>I_{3 p n}$. Hence, the MGRCMSE (or MGMSE) algorithm analysis result with multi-variate generalized coarse-grained structure is closer to the actual situation.

Lastly, compared with the MMSE, MGMSE, and MRCMSE, the proposed MGRCMSE can not only obtain smooth and easy-distinguishable entropy curves but also provide the analysis results for the actual composite situation. Thus, significant improvements were identified when using MGRCMSE when measuring the complexity of multi-channel signals.

\section{The proposed intelligent rotating machinery fault diagnosis method}

In this section, a novel intelligent fault diagnosis method for rotating machinery is proposed. Following the proposal, related algorithms such as SIM and HHO-SVM are described in detail.

\section{1 The proposed fault diagnosis scheme}

The authors aimed to provide a precise and reliable rotating machinery fault diagnosis process under different working conditions (i.e., a robust process). Thus, an intelligent multi-channel fault diagnosis method for the rotating machinery is proposed, primarily to enable the multi-channel signal fault diagnosis by using MGRCMSE, SIM, and HHO-SVM. The flowchart of the proposed method is shown in Fig. 6.

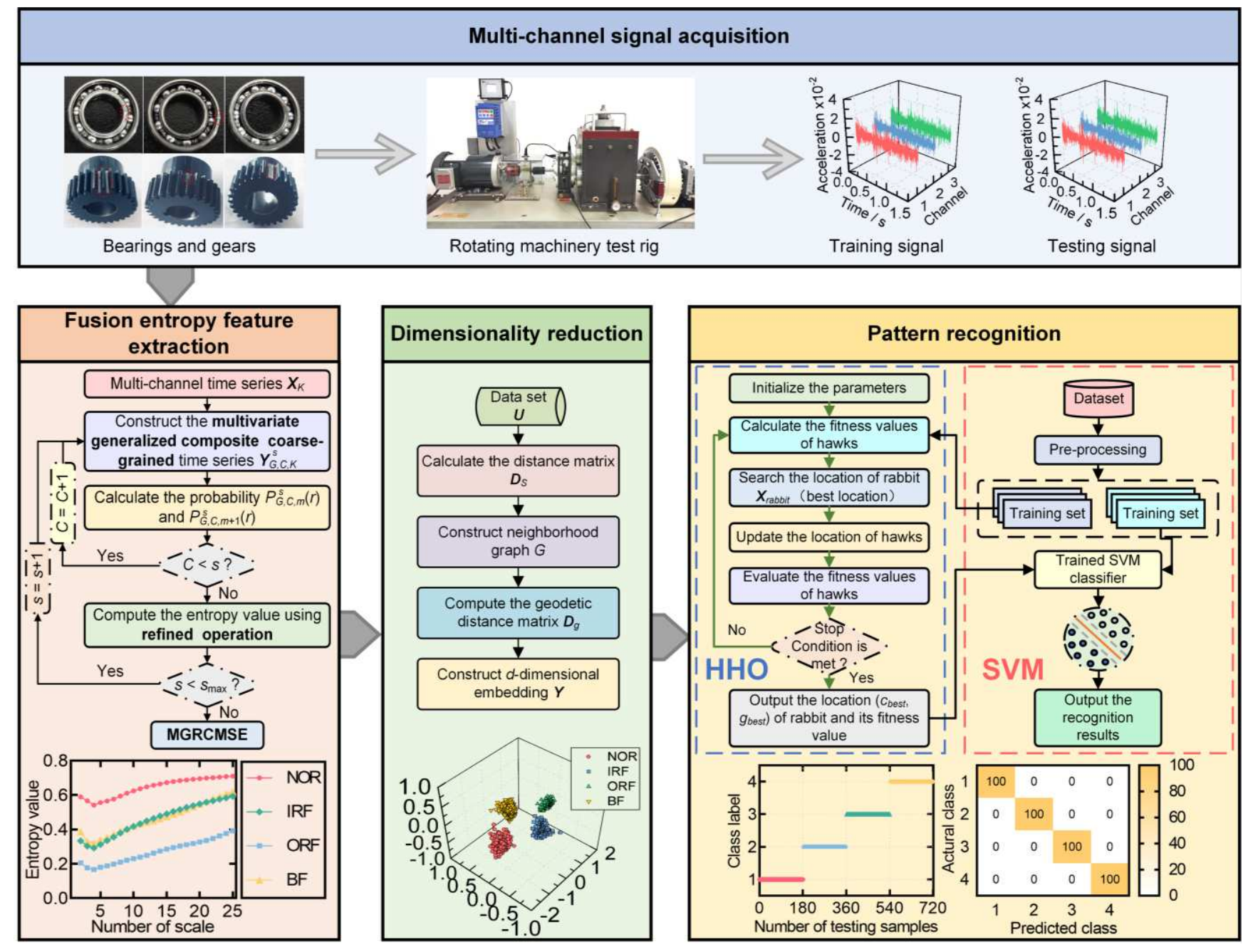

Fig. 6 Flowchart of the proposed intelligent multi-channel fault diagnosis model 
The specific steps needed to carry out the process are summarized as follows:

(1) Multi-channel signal acquisition. The multi-channel vibration acceleration signals of rotating machinery are collected through multiple sensors and in various working states. Then, the collected samples are randomly divided into training samples (10\%) and testing samples (90\%).

(2) Fusion entropy feature extraction. The proposed MGRCMSE method is used to extract fusion entropy features from multi-channel signals, allowing the extraction of a non-linear and high-dimensional fusion feature set.

(3) Dimensionality reduction. The SIM algorithm is introduced to map the MGRCMSE set to the low-dimensional space, obtaining a low-dimensional and sensitive feature set.

(4) Pattern recognition. The training feature set and testing feature set are normalized to the interval $[0,1]$, respectively. The training set is used as input to the HHO-SVM, allowing it to search for the optimal parameters $\left(c_{b e s t}, g_{b e s t}\right)$, allowing the authors to obtain the optimized SVM model. Finally, the testing set is input to the trained SVM classifier to diagnose faults.

\section{2 Supervised Isomap manifold learning method}

In the next step, the SIM algorithm is utilized to reduce the MGRCMSE feature set dimensionality, aiming to construct the sensitive, low-dimensional, and high-distinguish feature set. For a given data set $\boldsymbol{U}=\left\{\boldsymbol{u}_{i}\right\}_{i=1}^{N}$, the key SIM procedures are as follows:

(1) First, the supervised distance matrix $\boldsymbol{D}_{s}=\left\{d_{s}\left(\boldsymbol{u}_{i}, \boldsymbol{u}_{j}\right)\right\}$ is calculated:

$$
d_{s}\left(\boldsymbol{u}_{i}, \boldsymbol{u}_{j}\right)=\left\{\begin{array}{l}
\sqrt{1-\exp \frac{-d^{2}\left(\boldsymbol{u}_{i}, \boldsymbol{u}_{j}\right)}{\sigma}}, L \boldsymbol{u}_{i}=L \boldsymbol{u}_{j} \\
\sqrt{\exp \frac{d^{2}\left(\boldsymbol{u}_{i}, \boldsymbol{u}_{j}\right)}{\sigma}}-\eta, L \boldsymbol{u}_{i} \neq L \boldsymbol{u}_{j}
\end{array}\right.
$$

where $d\left(\boldsymbol{u}_{i}, \boldsymbol{u}_{j}\right)$ is the Euclidean distance between $\boldsymbol{u}_{i}$ and $\boldsymbol{u}_{j}, L \boldsymbol{u}_{i}$ is the $\boldsymbol{u}_{i}$ category, $\sigma$ is the average value of $d\left(\boldsymbol{u}_{i}, \boldsymbol{u}_{j}\right)$, and $\eta$ is the weighted coefficient.

(2) The neighborhood graph is constructed using the $k$-nearest neighbor method. If two sample points (e. g., $\boldsymbol{u}_{i}$ and $\boldsymbol{u}_{j}$ ) are adjacent to each other, there is a side connection with a side length of $d_{S}\left(\boldsymbol{u}_{i}, \boldsymbol{u}_{j}\right)$; otherwise, there is no side connection.

(3) The shortest path between any two samples can be calculated using the Dijkstra algorithm; the path is approximately regarded as the geodesic distance between these samples.

(4) The geodesic distance matrix is mapped to the low-dimensional space by using the multi-dimensional scaling algorithm [28], allowing us to obtain the final dimensionality reduction result.

\section{3 Harris hawks optimization-based support vector machine}

SVM, which is based on statistical learning theory, is the most commonly used classifier. It can not only deal with small samples and non-linear classification problems but also has good generalization ability. However, its performance is closely related to two important parameters $-c$ and $g$. Thus, to improve the performance of SVM, this section introduces the HHO-SVM classifier obtained after using the novel HHO algorithm to find the optimal values of SVM parameters. The HHO-SVM is a novel classifier (flowchart shown in Fig. 7) utilizing the Harris hawks optimization algorithm (for more details, see [29]) to optimize the key SVM parameters. The specific steps are shown next. 


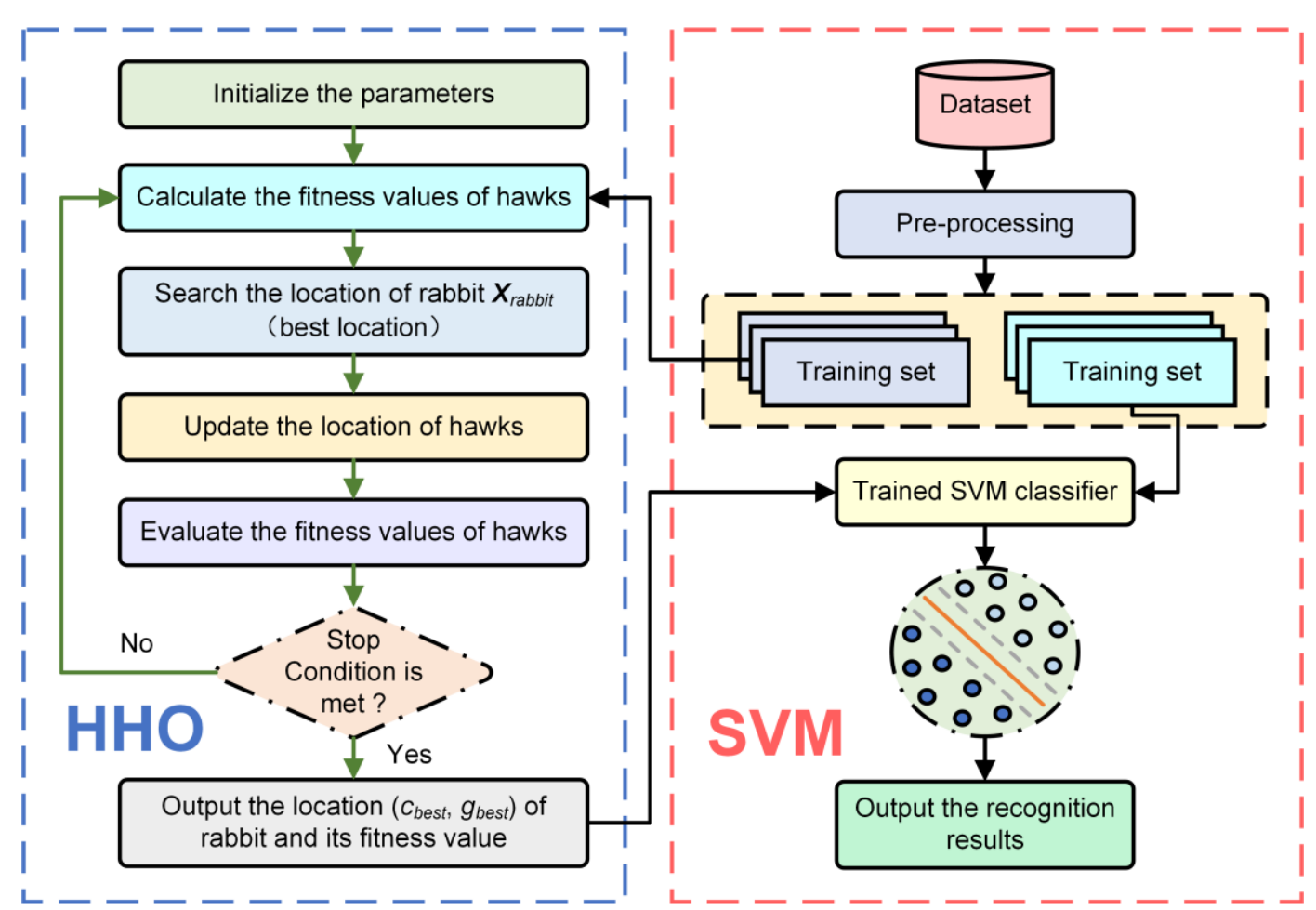

Fig. 7 The HHO-SVM flowchart

(1) Pre-process the data. The Eq. (16) is applied to normalize the input dataset between $[0,1]$.

$$
v^{\prime}=\left(v-v_{\min }\right) /\left(v_{\max }-v_{\min }\right)
$$

where $v$ and $v^{\prime}$ are the values before and after normalization, respectively. Similarly, $v_{\max }$ and $v_{\min }$ are the maximum and minimum values, respectively.

(2) Initialize the parameters. The initial HHO population size $\left(N_{p}\right)$ is set to 20 , and the maximum number of iterations $(T)$ is 100 . For the optimization problem at hand, the main purpose is to find the optimal SVM parameter values $-c$ and $g$. The position of each Harris hawks is defined using $\boldsymbol{H}_{i}=(c, g)$, with the lower and upper position boundaries set as $\boldsymbol{L} \boldsymbol{B}=[0.001,0.001]$ and $\boldsymbol{U} \boldsymbol{B}=[100,100]$, respectively.

(3) Calculate the fitness values. To better evaluate the quality of each Harris hawk, the average training sample error classification rate after a three-fold crossover is defined as the fitness value. In other words, the training samples are divided into three subsets; each subset is regarded as a verification set, while the two remainings are used as training sets. Finally, the average error classification rate of the three groups is taken as the fitness value.

(4) Search the Prey location. The best Harris hawk position, which corresponds to the best fitness value, is considered as the prey location $\left(\boldsymbol{H}_{\text {rabbit }}\right)$ under the current iteration $t$.

(5) Update the prey escaping energy and jump strength. The escaping energy $E$ and the jump strength $J$ of the prey can be updated, using Eq. (17):

$$
\begin{aligned}
& E=2 E_{0}(1-t / T) \\
& J=2\left(1-r_{1}\right) \\
& E_{0}=2 r_{2}-1
\end{aligned}
$$

where, $r_{1}$ and $r_{2}$ are random numbers between $(0,1)$.

(6) Update the Harris hawk locations. The position of each Harris hawk can be updated by using the Eqs. (18) to (22). If the fitness value of the new position is lower than that of the previous position, the previous position is replaced with the new one; otherwise, the previous position is retained. 
1) Survey phase (i.e., $|E| \geq 1)$. The position of each Harris hawks is updated by:

$$
\boldsymbol{H}(t+1)= \begin{cases}\boldsymbol{H}_{\text {rand }}(t)-r_{3}\left|\boldsymbol{H}_{\text {rand }}(t)-2 r_{4} \boldsymbol{H}(t+1)\right| & q \geq 0.5 \\ \left(\boldsymbol{H}_{\text {rabbit }}(t)-\boldsymbol{H}_{m}(t)\right)-r_{5}\left(L B+r_{6}(\boldsymbol{U B}-\boldsymbol{L B})\right) & q<0.5\end{cases}
$$

where $\boldsymbol{H}(t)$ and $\boldsymbol{H}(t+1)$ are the Harris hawk positions at $t$ and $t+1$ iteration, respectively. $\boldsymbol{H}_{\text {rabbit }}(t)$ is the prey location, $\boldsymbol{H}_{\text {rand }}(t)$ is the random Harris hawk position in the current iteration, $\boldsymbol{H}_{m}(t)=\left[\sum_{i=1}^{N_{p}} \boldsymbol{H}_{i}(t)\right] / N_{p}$ is the average position of all Harris hawks positions in the current iteration, while $r_{3}, r_{4}, r_{5}, r_{6}$, and $q$ are the random numbers between 0 and 1 .

2) Development phase (i.e., $0 \leq|E|<1$ ). The variable $r_{7}$ is defined as the probability of the prey escaping and is a random number between 0 and 1 . For example, $r_{7}<0.5$ indicates that the prey has succeeded in escaping while $r_{7} \geq 0.5$ indicates that it has failed. This phase can be further into four possible situations:

- Soft besiege (i.e., $r_{7} \geq 0.5, \mid E \geq 0.5$ ) - the position of each Harris hawk is updated by:

$$
\begin{gathered}
\boldsymbol{H}(t+1)=\Delta \boldsymbol{H}(t)-E\left|J \boldsymbol{H}_{\text {rabbit }}(t)-\boldsymbol{H}(t)\right| \\
\Delta \boldsymbol{H}(t)=\boldsymbol{H}_{\text {rabbit }}(t)-\boldsymbol{H}(t)
\end{gathered}
$$

- Hard besiege (i.e., $r_{7} \geq 0.5,0 \leq|E|<0.5$ ) - the position of each Harris hawk is updated by:

$$
\boldsymbol{H}(t+1)=\boldsymbol{H}_{\text {rabbit }}(t)-E|\Delta \boldsymbol{H}(t)|
$$

- Soft besiege with progressive rapid dives, (i.e., $r_{7}<0.5,|E| \geq 0.5$ ) - the position of each Harris hawk is updated by:

$$
\begin{aligned}
& \boldsymbol{H}(t+1)= \begin{cases}\boldsymbol{Y}_{H}=\boldsymbol{H}_{\text {rabbii }}(t)-E\left|J \boldsymbol{H}_{\text {rabbit }}(t)-\boldsymbol{H}(t)\right| & \text { if } F\left(\boldsymbol{Y}_{H}\right)<F(\boldsymbol{H}(t)) \\
\boldsymbol{Z}_{H}=\boldsymbol{Y}_{H}+\boldsymbol{S} \times L F(D) & \text { if } F\left(\boldsymbol{Z}_{H}\right)<F(\boldsymbol{H}(t))\end{cases} \\
& L F(x)=\frac{0.01 \times \mu \times \theta}{|\gamma|^{1 / \beta}}, \theta=\left(\left[\Gamma(1+\beta) \times \sin \left(\frac{\pi \beta}{2}\right)\right] /\left[\Gamma\left(\frac{1+\beta}{2}\right) \times \beta \times 2^{\left(\frac{\beta-1}{2}\right)}\right]\right)^{1 / \beta}
\end{aligned}
$$

where $D=2$ is the positional dimension, $S$ is a random vector of size $1 \times D, L F(x)$ is the Levy flight function, and $\mu, \gamma$ are the random number between 0 and 1. Finally, $\beta=1.5$ and $\Gamma(\cdot)$ is a Gamma function.

- Hard besiege with progressive rapid dives, (i. e., $r_{7}<0.5,|E|<0.5$ ) - the position of each Harris hawk is updated by:

$$
\boldsymbol{H}(t+1)= \begin{cases}\boldsymbol{Y}_{H}^{\prime}=\boldsymbol{H}_{\text {rabbit }}(t)-E\left|J \boldsymbol{H}_{\text {rabbit }}(t)-\boldsymbol{H}_{m}(t)\right| & \text { if } F\left(\boldsymbol{Y}_{H}^{\prime}\right)<F(\boldsymbol{H}(t)) \\ \boldsymbol{Z}_{H}^{\prime}=\boldsymbol{Y}_{H}^{\prime}+\boldsymbol{S} \times L F(D) & \text { if } F\left(\boldsymbol{Z}_{H}^{\prime}\right)<F(\boldsymbol{H}(t))\end{cases}
$$

(7) Judge whether the stop condition is met. If the simulation has reached the previously selected maximum number of iterations, the cycle is terminated. The final prey position $\left(c_{\text {best }}, g_{\text {best }}\right)$ is used as output. Otherwise, step (2) is repeated until the stop criterion is met.

(8) Construct the optimal SVM training model. The final prey position is implemented in the SVM prediction model.

(9) Identify the testing set categories. The testing set samples are input into the trained SVM prediction model for classification with the recognition results as outputs.

\section{Experimental verification}

The experiments were carried out using two case studies as examples, the first being concerned with bearings and the second with gears. The aim was to evaluate the performance and verify the effectiveness of the proposed 
fault diagnosis method. Moreover, several comparative experiments were carried out to evaluate the proposed method and verify its advantages. These include both the comparison with other multi-channel feature extraction methods and the comparison with several single-channel feature extraction methods.

\section{1 Experimental rig}

The experimental data were collected from the drivetrain dynamics simulator (Fig. 8). The experimental platform consists of the variable speed drive motor, programmable motor controller, planetary gearbox, parallel shaft gearbox, brake, and programmable magnetic controller. The planetary gearbox parameters are shown in Table 2.

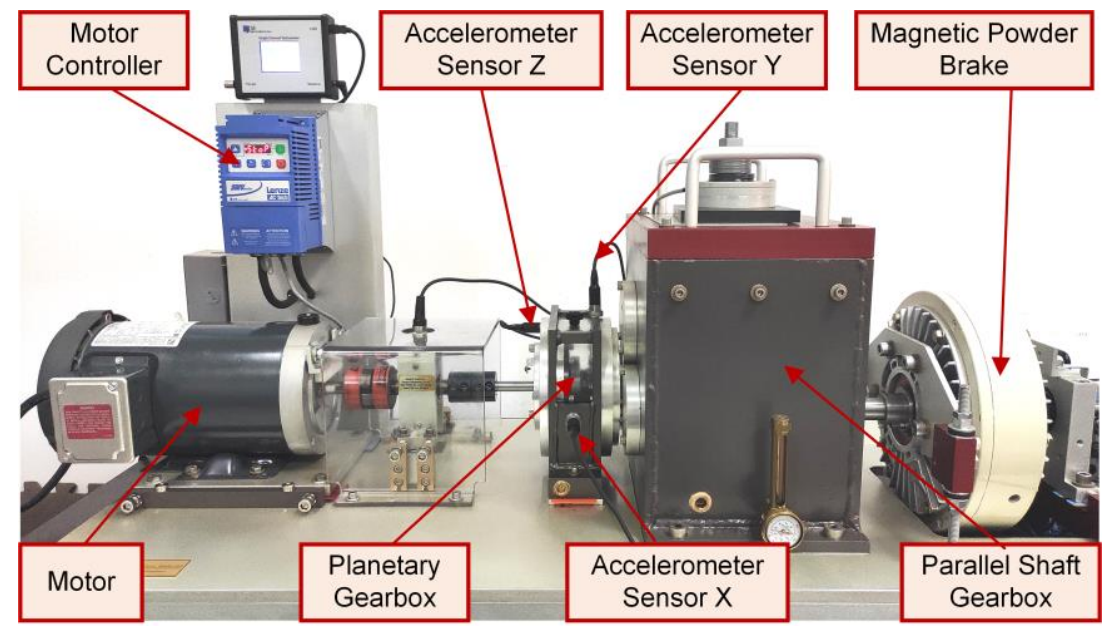

Fig. 8 Experimental test platform

Table 2 Main parameters of planetary gearbox

\begin{tabular}{lll}
\hline Component & Number of gear teeth & Second stage \\
\cline { 2 - 3 } & First stage & 100 \\
\hline Gear ring & 100 & 36 (the number of planetary gears is 4) \\
Planet gear & 40 (the number of planetary gears is 3) & 28 \\
Sun gear & 20 & \\
\hline
\end{tabular}

\section{2 Case study 1: Rolling bearing fault diagnosis}

\section{2. 1 Rolling bearing data set}

Four bearing operation states were considered in this experiment: normal, inner ring fault, outer ring fault, and ball fault, with each being outlined in Fig. 9. The working experimental rig conditions were selected as follows: the driving speed was set to $25 \mathrm{~Hz}$ and the load to $0 \mathrm{Nm}$. The acceleration sensors were used to collect the vibration signals in the $\mathrm{X}, \mathrm{Y}$, and $\mathrm{Z}$ direction, each obtained with the sampling frequency of $3000 \mathrm{~Hz}$ (please see Table 3). Finally, the three-channel time-domain bearing waveforms under various operation states are shown in Fig. 10.

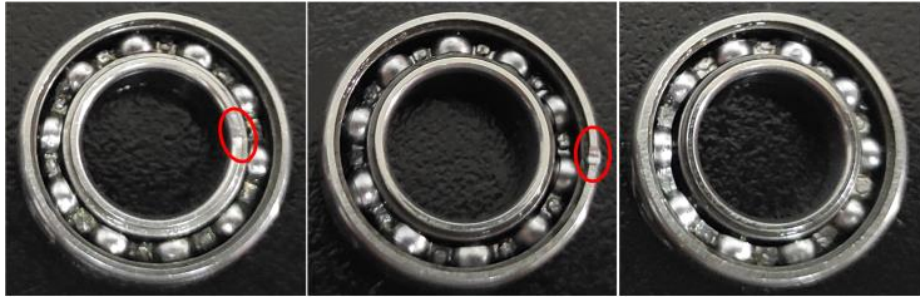

(a) (b) (c)

Fig. 9 Bearing faults: (a) inner ring fault, (b) outer ring fault, and (c) ball fault 
Table 3 Description of rolling bearings under four working states

\begin{tabular}{|c|c|c|c|c|c|c|c|c|}
\hline State & $\begin{array}{l}\text { Abbre } \\
\text { viation }\end{array}$ & Description & Class & Channel & $\begin{array}{l}\text { Data } \\
\text { points }\end{array}$ & $\begin{array}{l}\text { Number of } \\
\text { samples }\end{array}$ & $\begin{array}{l}\text { Number of } \\
\text { training } \\
\text { samples }\end{array}$ & $\begin{array}{l}\text { Number of } \\
\text { testing } \\
\text { samples }\end{array}$ \\
\hline \multirow[t]{3}{*}{ Normal } & \multirow[t]{3}{*}{ NOR } & \multirow[t]{3}{*}{ No fault } & \multirow[t]{3}{*}{1} & $X$ & 3000 & \multirow[t]{3}{*}{200} & \multirow[t]{3}{*}{20} & \multirow[t]{3}{*}{180} \\
\hline & & & & $\mathrm{Y}$ & 3000 & & & \\
\hline & & & & $\mathrm{Z}$ & 3000 & & & \\
\hline \multirow{3}{*}{$\begin{array}{l}\text { Inner ring } \\
\text { fault }\end{array}$} & \multirow[t]{3}{*}{ IRF } & \multirow{3}{*}{$\begin{array}{l}\text { A fault occurs } \\
\text { in the inner } \\
\text { ring }\end{array}$} & \multirow[t]{3}{*}{2} & $\mathrm{X}$ & 3000 & \multirow[t]{3}{*}{200} & \multirow[t]{3}{*}{20} & \multirow[t]{3}{*}{180} \\
\hline & & & & $\mathrm{Y}$ & 3000 & & & \\
\hline & & & & $\mathrm{Z}$ & 3000 & & & \\
\hline \multirow{3}{*}{$\begin{array}{l}\text { Outer } \\
\text { ring fault }\end{array}$} & \multirow[t]{3}{*}{ ORF } & \multirow{3}{*}{$\begin{array}{l}\text { A fault occurs } \\
\text { in the outer } \\
\text { ring }\end{array}$} & \multirow[t]{3}{*}{3} & $\mathrm{X}$ & 3000 & \multirow[t]{3}{*}{200} & \multirow[t]{3}{*}{20} & \multirow[t]{3}{*}{180} \\
\hline & & & & $\mathrm{Y}$ & 3000 & & & \\
\hline & & & & $\mathrm{Z}$ & 3000 & & & \\
\hline \multirow[t]{3}{*}{ Ball fault } & \multirow[t]{3}{*}{$\mathrm{BF}$} & \multirow{3}{*}{$\begin{array}{l}\text { A fault occurs } \\
\text { in the ball }\end{array}$} & \multirow[t]{3}{*}{4} & $\mathrm{X}$ & 3000 & \multirow[t]{3}{*}{200} & \multirow[t]{3}{*}{20} & \multirow[t]{3}{*}{180} \\
\hline & & & & Y & 3000 & & & \\
\hline & & & & $\mathrm{Z}$ & 3000 & & & \\
\hline
\end{tabular}

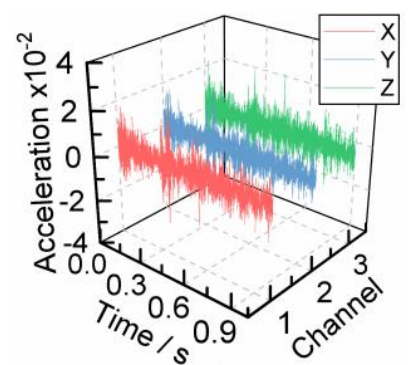

(a)

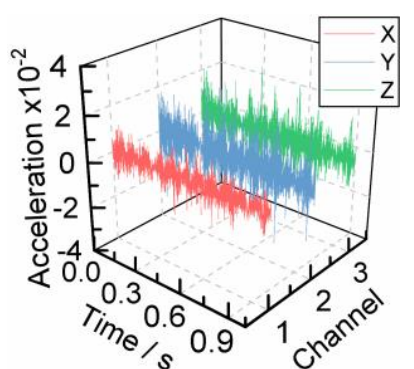

(b)

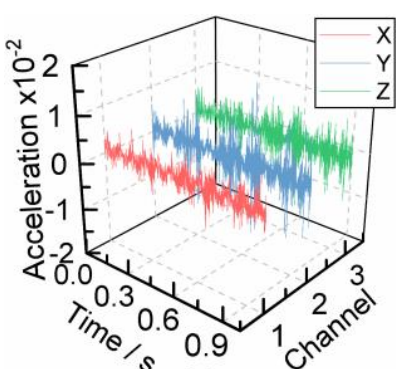

(c)

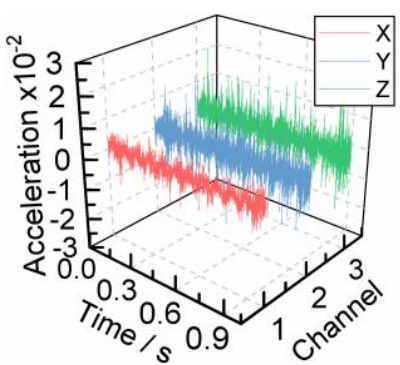

(d)

Fig. 10 Time-domain waveforms of rolling bearing vibration signals: (a) NOR, (b) IRF, (c) ORF, and (d) BF

\section{2. 2 Experimental results and analysis}

According to the proposed fault diagnosis process, the proposed MGRCMSE method is applied to extract the fusion features from three-channel bearing signals. The results for a total of 800 analyzed bearing samples under four different states are plotted in Fig. 11.
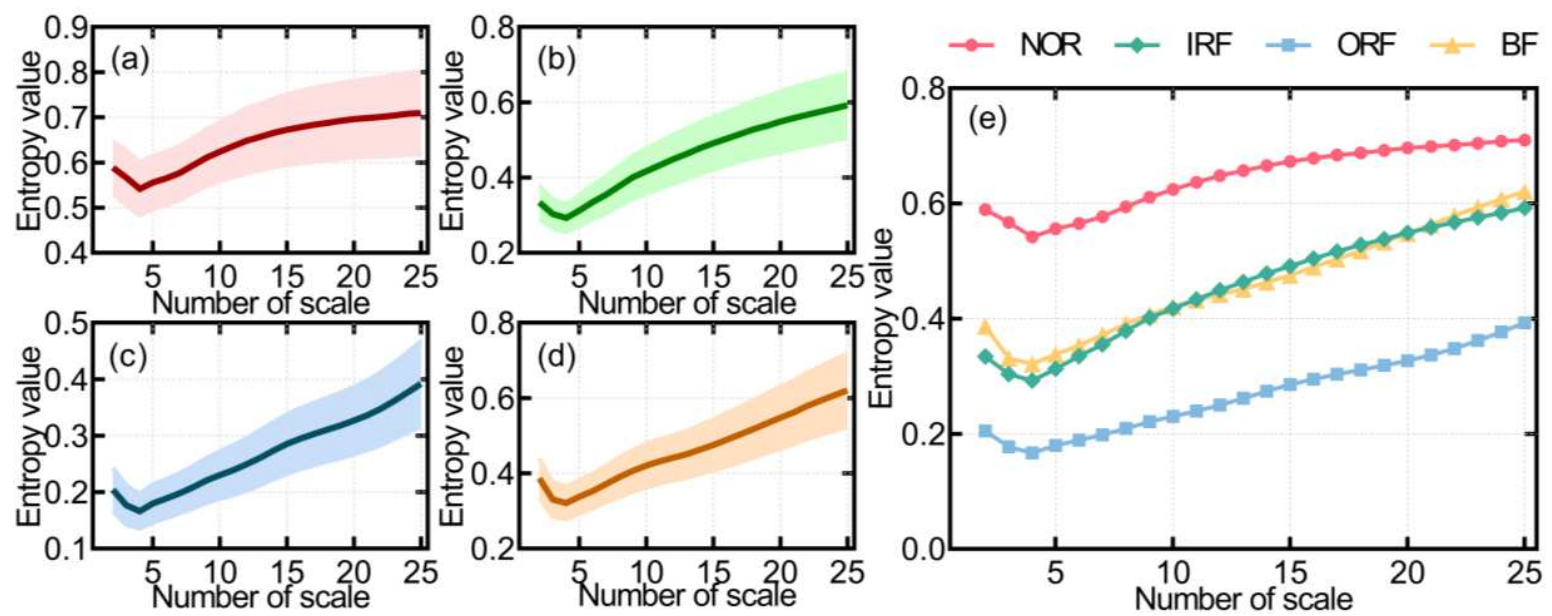

Fig. 11 The MGRCMSE entropy curves: (a) NOR, (b) IRF, (c) ORF, (d) BF, and (e) mean MGRCMSE curves

Several phenomena were found in Fig. 11. Firstly, the mean entropy values of the normal bearing signals are higher when compared to the fault bearing signals under, regardless of the scale. Such behavior is caused by the self-similarity of normal signals, which is weaker than that of fault signals. Therefore, the normal state entropy 
values are higher than their fault state counterparts. Hence, the MGRCMSE algorithm can effectively monitor the occurrence of bearing failure. Secondly, the entropy values of the four operation states at most scales are ordered as follows $E_{N O R}>E_{B F}>E_{I R F}>E_{O R F}$, which is consistent with the realistic case. It can be concluded that both phenomena confirm that the MGRCMSE algorithm can generally identify the faults.

However, it is apparent from Fig. 11 that the IRF and BF entropy mean curves are rather similar. Moreover, the extracted feature set is high-dimensional and redundant; if directly input to the classifier as such, both the recognition time and accuracy will be increased. For this reason, according to the proposed fault diagnosis method, a supervised manifold learning algorithm (SIM) is adopted to generate the corresponding low-dimensional set. The results are shown in Fig. 12. It should be pointed out that based on several experiments, the optimal SIM parameters are $d=3, k=92$, and $\eta=0.4$.

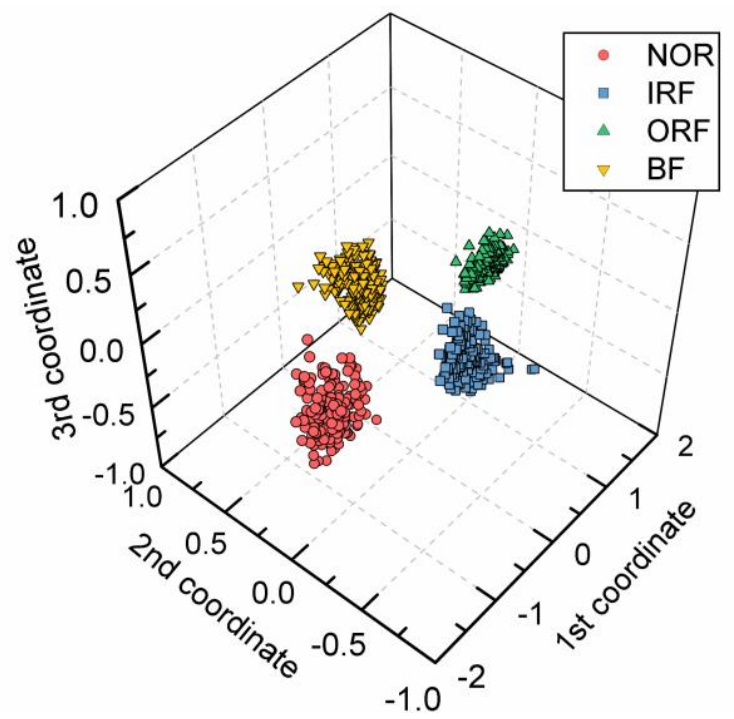

Fig.12 The 3D result obtained using the SIM

In the 3D visualization results (see Fig. 12), it was shown that SIM could entirely separate the four types of samples. Additionally, it is evident that samples are grouped based on their state. This proves that the SIM algorithm can effectively obtain a low-dimensional and sensitive set, which is easily-distinguishable.

When classifying the set, the above-presented fusion entropy-manifold (i.e., MGRCMSE+SIM) feature set is used as input for the HHO-SVM classifier. The HHO algorithm is first used to find the optimal SVM parameters (i.e., $c_{\text {best }}=79.86, g_{\text {best }}=7.62$ ), allowing us to establish the corresponding optimal SVM prediction model. The training stage fitness curve is shown in Fig. 13 (a). Testing samples are input to the previously trained SVM classifier for pattern recognition; output results and the confusion matrix of testing samples are shown in Figs. 13 (b) and (c), respectively.

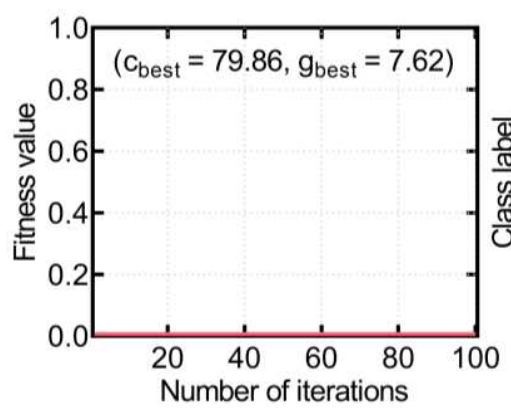

(a)

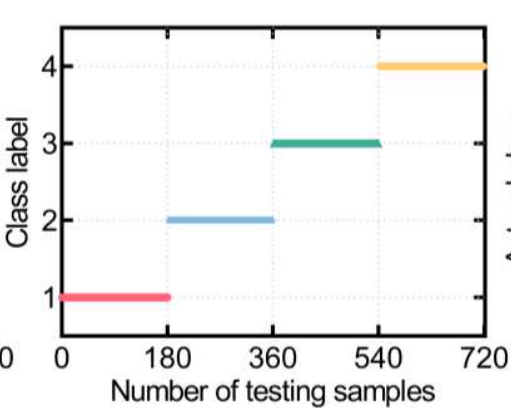

(b)

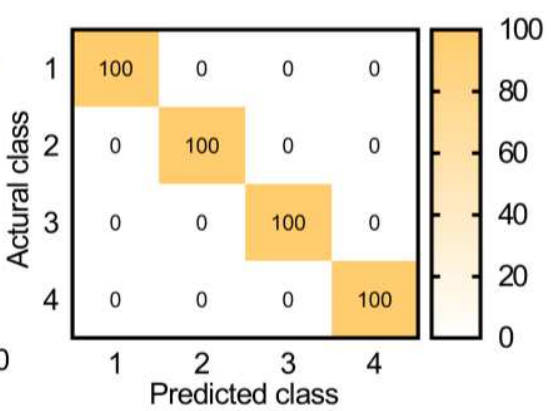

(c)

Fig. 13 Fault diagnosis results: (a) fitness curve, (b) output results, and (c) confusion matrix (\%) 
As shown in Fig 13, 720 test samples were correctly identified with an average recognition rate of 100\%. More importantly, it was proven that the proposed fault diagnosis method could effectively and accurately diagnose bearings in different states.

\section{3 Case study 2: Gear fault diagnosis}

\section{3. 1 Gear data set}

In this experiment, four gear operation states were established: normal, tooth breakage fault, missing tooth fault, and tooth root fault (Fig. 14). The experimental working conditions were as follows: the driving speed of $25 \mathrm{~Hz}$, and the load is $0 \mathrm{Nm}$. Three acceleration sensors were used to collect the vibration signals in $\mathrm{X}$, Y, and Z directions under different operation modes, obtained at the sampling frequency of $3000 \mathrm{~Hz}$. The detailed description is shown in Table 4, while the three-channel gear waveforms under each operation mode are plotted in Fig. 15.

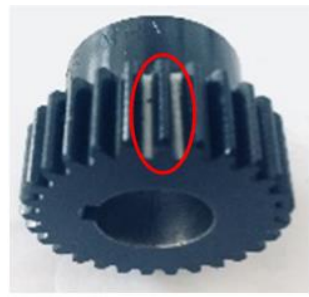

(a)

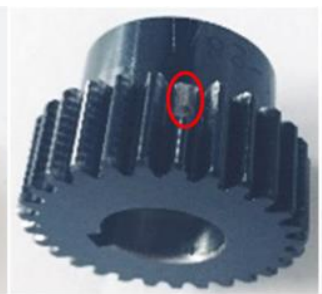

(b)

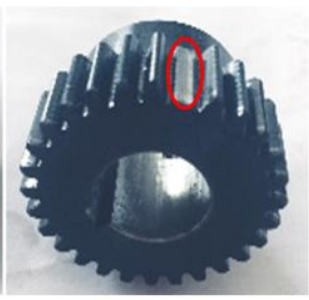

(c)

Fig. 14 Gear faults: (a) tooth root fault, (b) tooth breakage fault, and (c) missing tooth fault

Table 4

Description of gears under four working states

\begin{tabular}{|c|c|c|c|c|c|c|c|c|}
\hline State & $\begin{array}{l}\text { Abbre } \\
\text { viation }\end{array}$ & Description & Class & Channel & $\begin{array}{l}\text { Data } \\
\text { points }\end{array}$ & $\begin{array}{l}\text { Number of } \\
\text { samples }\end{array}$ & $\begin{array}{l}\text { Number of } \\
\text { training } \\
\text { samples }\end{array}$ & $\begin{array}{l}\text { Number of } \\
\text { testing } \\
\text { samples }\end{array}$ \\
\hline \multirow[t]{3}{*}{ Normal } & \multirow[t]{3}{*}{ NOR } & \multirow[t]{3}{*}{ Healthy } & \multirow[t]{3}{*}{1} & $X$ & 3000 & \multirow[t]{3}{*}{200} & \multirow[t]{3}{*}{20} & \multirow[t]{3}{*}{180} \\
\hline & & & & $\mathrm{Y}$ & 3000 & & & \\
\hline & & & & $\mathrm{Z}$ & 3000 & & & \\
\hline \multirow{3}{*}{$\begin{array}{l}\text { Tooth } \\
\text { breakage } \\
\text { fault }\end{array}$} & \multirow[t]{3}{*}{$\mathrm{TBF}$} & \multirow{3}{*}{$\begin{array}{l}\text { Signal tooth } \\
\text { breakage }\end{array}$} & \multirow[t]{3}{*}{2} & $X$ & 3000 & \multirow[t]{3}{*}{200} & \multirow[t]{3}{*}{20} & \multirow[t]{3}{*}{180} \\
\hline & & & & $\mathrm{Y}$ & 3000 & & & \\
\hline & & & & $\mathrm{Z}$ & 3000 & & & \\
\hline \multirow{3}{*}{$\begin{array}{l}\text { Missing } \\
\text { tooth } \\
\text { fault }\end{array}$} & \multirow[t]{3}{*}{ MTF } & \multirow{3}{*}{$\begin{array}{l}\text { Signal tooth } \\
\text { missing }\end{array}$} & \multirow[t]{3}{*}{3} & $X$ & 3000 & \multirow[t]{3}{*}{200} & \multirow[t]{3}{*}{20} & \multirow[t]{3}{*}{180} \\
\hline & & & & $Y$ & 3000 & & & \\
\hline & & & & $\mathrm{Z}$ & 3000 & & & \\
\hline \multirow{3}{*}{$\begin{array}{l}\text { Tooth } \\
\text { root fault }\end{array}$} & \multirow[t]{3}{*}{ TRF } & \multirow{3}{*}{$\begin{array}{l}\text { A fault occurs } \\
\text { in the gear } \\
\text { tooth root }\end{array}$} & \multirow[t]{3}{*}{4} & $X$ & 3000 & \multirow[t]{3}{*}{200} & \multirow[t]{3}{*}{20} & \multirow[t]{3}{*}{180} \\
\hline & & & & $\mathrm{Y}$ & 3000 & & & \\
\hline & & & & $\mathrm{Z}$ & 3000 & & & \\
\hline
\end{tabular}

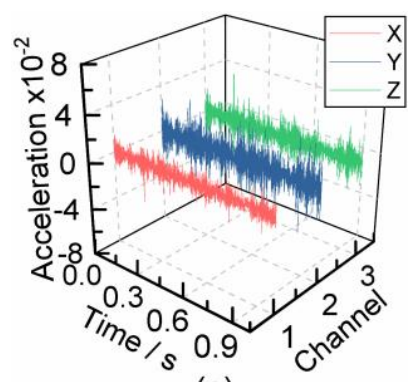

(a)

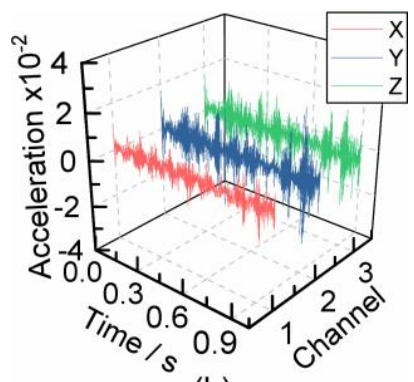

(b)

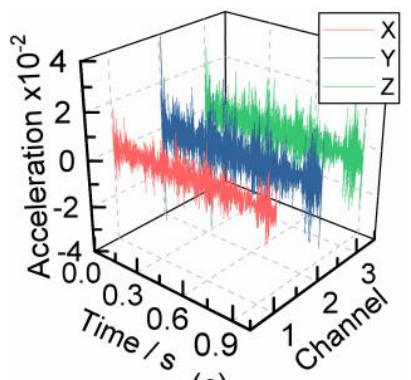

(c)

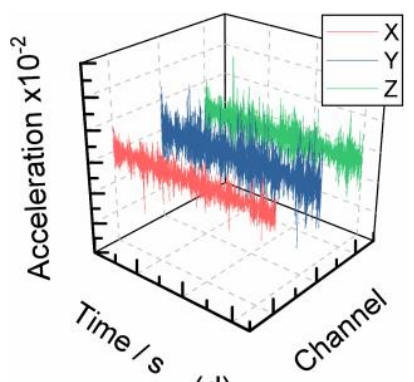

(d)

Fig. 15 Time-domain waveforms of three-channel gear vibration signals: (a) NOR, (b) TBF, (c) MTF, and (d) TRF 


\section{3. 2 Experimental results and analysis}

According to the proposed fault diagnosis method, the MGRCMSE algorithm was utilized to extract the fusion features from three-channel gear signals, as shown in Fig. 16.
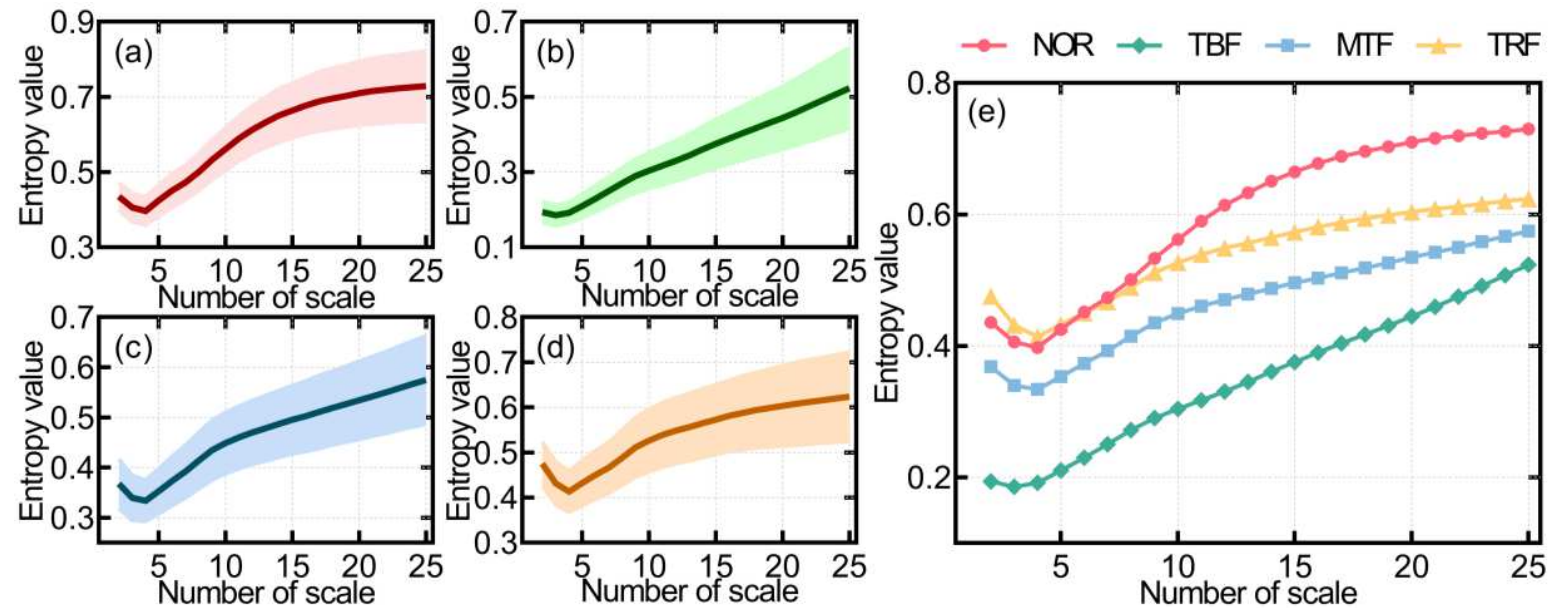

Fig. 16 MGRCMSE entropy curves: (a) NOR, (b) TBF, (c) MTF, (d) TRF, and (e) mean MGRCMSE curves

The following observations were made based on Fig. 16: (1) the mean entropy values of normal gear signals were higher than that of faulty gear signals in most of the scales. The main reason for such behavior was the vibration signals in the normal state, which have shown strong randomness, while the vibration signals in faulty states had a prolonged impact. Thus, the entropy values of fault states were smaller than that of the normal state, indicating that the MGRCMSE can monitor the occurrence of gear failure. (2) The mean entropy curves of the gear signals under four states can be separated from each other, showing that the proposed method is able to effectively distinguish between the fault states.

The SIM was used to reduce the extracted MGRCMSE feature set dimensionality (the result is plotted in Fig. 17). It should be pointed out that the optimal SIM parameters were obtained experimentally and are as follows: $d=3$, $k=193$, and $\eta=0.38$.

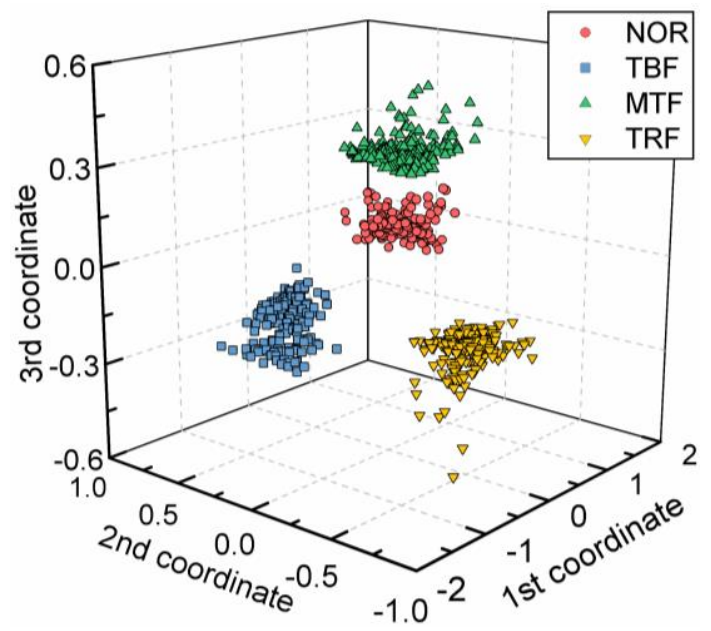

Fig. 17 The 3D result obtained using the SIM

The 3D visualization results (shown in Fig. 17) show that SIM thoroughly separates the four-state samples, completely avoiding sample overlap. Thus, the SIM effectiveness in the dimensionality reduction of the gear feature set is considered to be verified.

Furthermore, the obtained fusion entropy-manifold (i.e., MGRCMSE+SIM) feature set is used as input for the classification using HHO-SVM. The HHO algorithm is firstly employed to find the optimal SVM parameters $\left(c_{\text {best }}=\right.$ 
56.99, $g_{\text {best }}=24.84$ ), allowing us to establish the corresponding optimal SVM prediction model. The training stage fitness curve is shown in Fig. 18 (a), while the testing sample output results and the confusion matrix are shown in Figs. 18 (b) and (c).

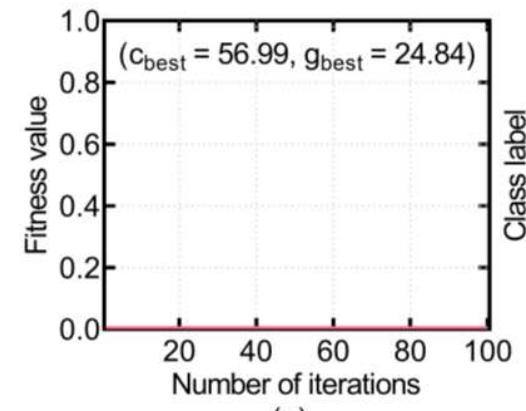

(a)

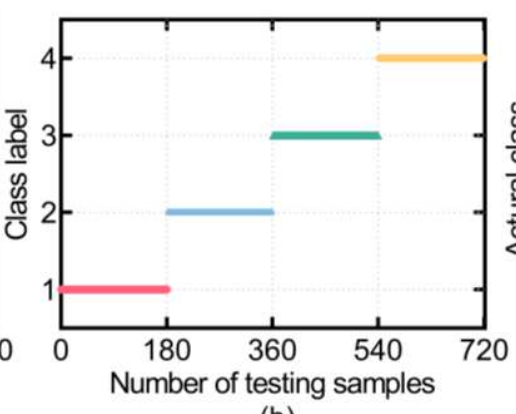

(b)

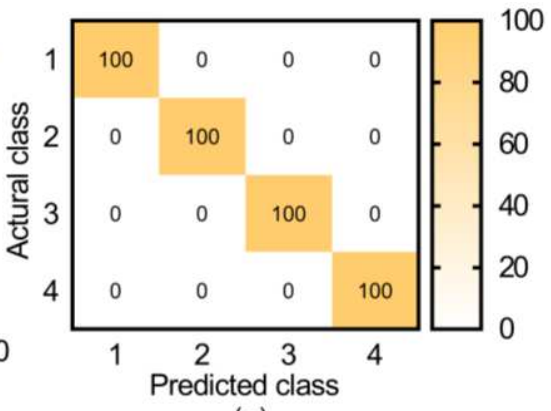

(c)

Fig. 18 Fault diagnosis results: (a) fitness curve, (b) output result, and (c) confusion matrix (\%)

It can be seen from Fig. 18 that the HHO-SVM correctly identified all the 720 testing samples. Moreover, the average recognition accuracy of the proposed method for the gear data set was thus $100 \%$, proving that it can effectively and accurately diagnose various gear operation states.

\section{4 Performance comparison}

The advantages of the proposed MGRCMSE method were then further explored using the bearing fault diagnosis as an example; two comparative studies were carried out.

(1) Comparison with other multi-channel feature extraction algorithm. In this section, the MGRCMSE algorithm performance was compared to the performance of the MGMSE algorithm; the results are shown in Fig. 19. The MGMSE algorithm parameters used in the study are listed in Table 5.

Table 5 Parameter settings for various feature extraction methods

\begin{tabular}{llll}
\hline Reference & Algorithms & Type & Parameter settings \\
\hline Yin et al. [26] & MGMSE & multi-channel method & $m=2, r=0.15 s d, N=3000$, and $s=25$ \\
Wang et al. (proposed) & MGRCMSE & multi-channel method & $m=2, r=0.15 s d, N=3000$, and $s=25$ \\
\hline
\end{tabular}

According to Figs. 19 (a-d), the mean MGMSE and MGRCMSE entropy curves for each bearing state are relatively similar. However, it is noted that the mean MGMSE entropy curves have slight fluctuations in large scales, especially for bearing without faults. Next, it can be seen in Figs. 19 (e-h) that the entropy values obtained using MGRCMSE have low standard deviations on most scales. Additionally, several random entropy curves were drawn for each bearing state signal, using both algorithms, as shown in Figs. 19 (i-1). When compared with the MGRCMSE, the MGMSE entropy curves have larger fluctuations. These phenomena prove that the proposed MGRCMSE algorithm can obtain more stable entropy results when compared to the MGMSE.

Furthermore, the feature sets obtained using the above-mentioned algorithms were input into the HHO-SVM for recognition (for results, please see Fig. 20).

As illustrated in Fig. 20, a total of 126 MGRCMSE testing samples were misclassified, including four NOR samples, 42 IRF samples, 13 ORF samples, and 67 BF samples. For a comparison, when using MGMSE, 148 testing samples were not correctly classified, including nine NOR samples, 61 IRF samples, six ORF samples, and 72 BF samples. Thus, compared to the MGRCMSE (the average recognition rate of 82.5\%), the average MGMSE recognition rate was 3.06\% lower (i.e., 79.44\%). Thus, it is verified that the MGRCMSE performance in feature extraction is an improvement when compared to MGMSE. 


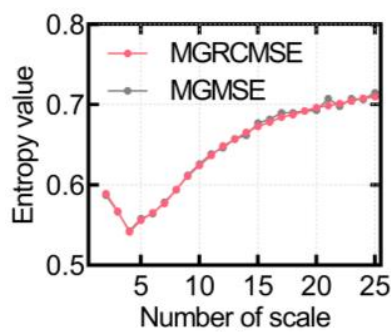

(a)

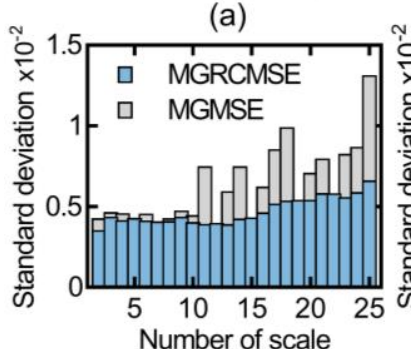

(e)

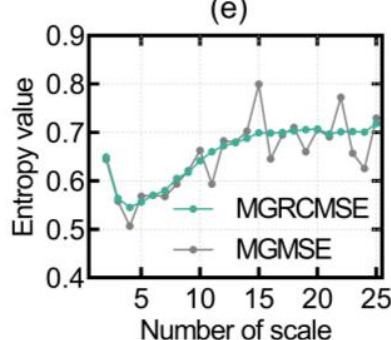

(i)

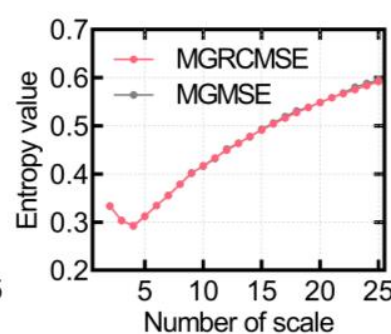

(b)

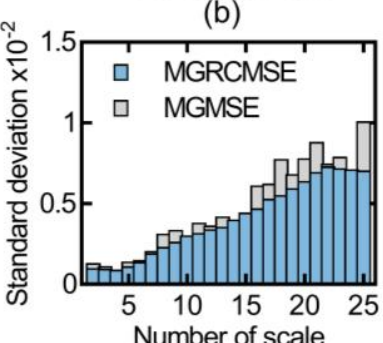

(f)

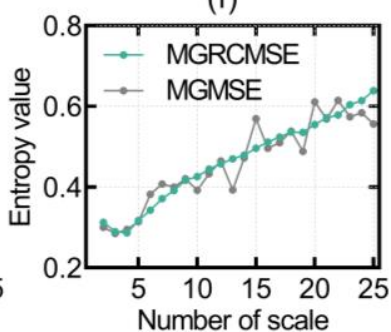

(j)

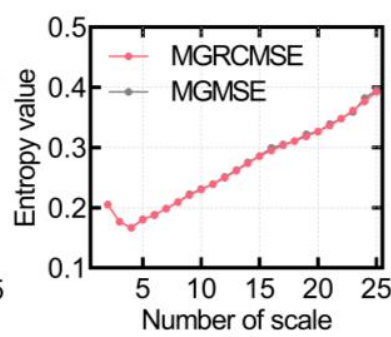

(c)

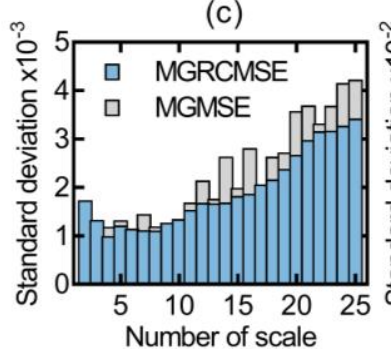

(g)

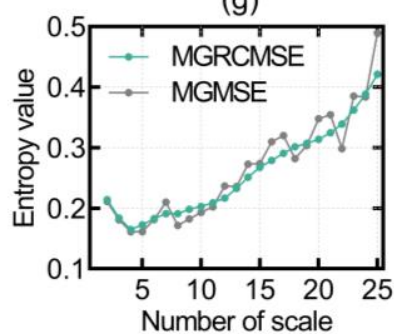

(k)

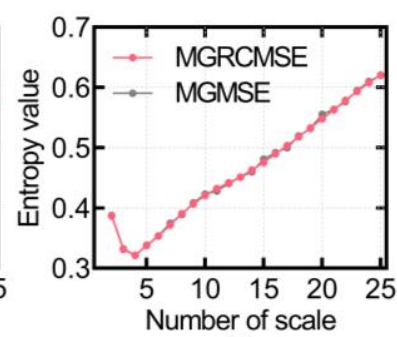

(d)

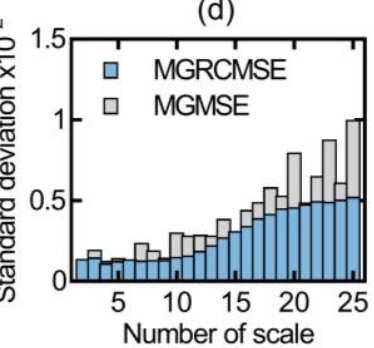

(h)

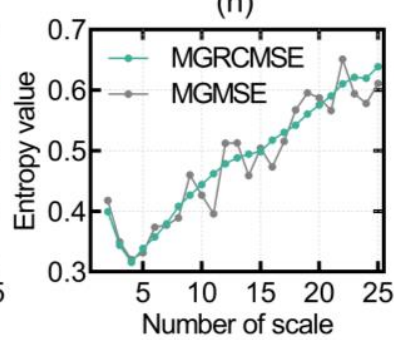

(I)

Fig. 19 Mean entropy curves, standard deviation values, and random group entropy curves, using two feature extraction algorithms. (a) NOR mean curves, (b) IRF mean curves (c) ORF mean curves, (d) BF mean curves, (e) NOR standard deviation, (f) IRF standard deviation, (g) ORF standard deviation, (h) BF standard deviation, (i) NOR entropy curves, (g) IRF entropy curves, (k) ORF entropy curves, and (1) BF entropy curves

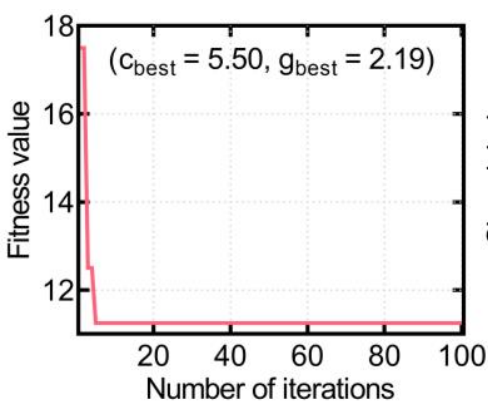

(a)

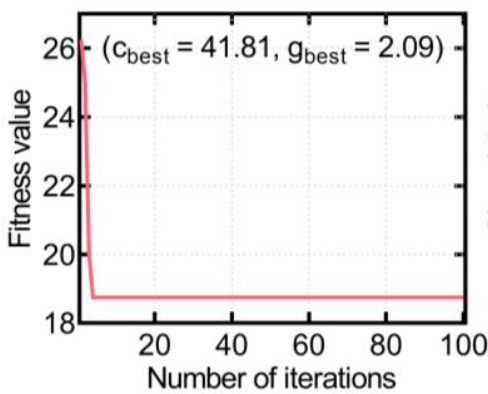

(d)

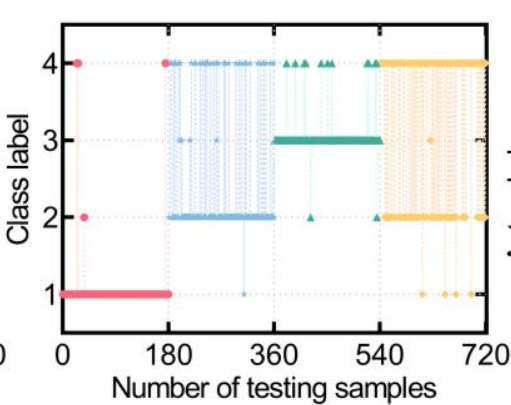

(b)

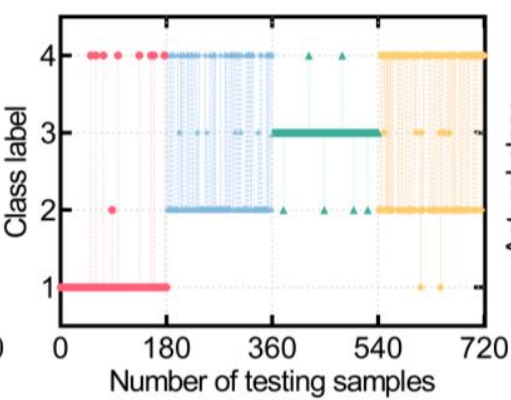

(e)

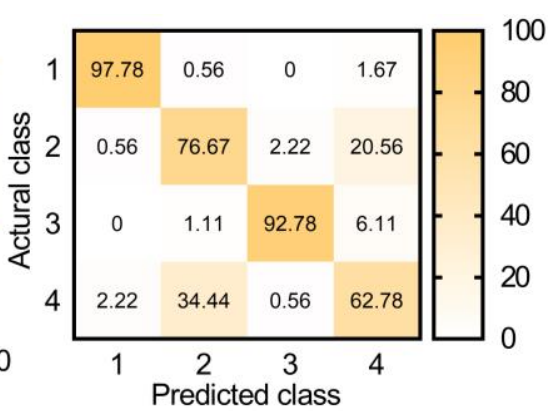

(c)

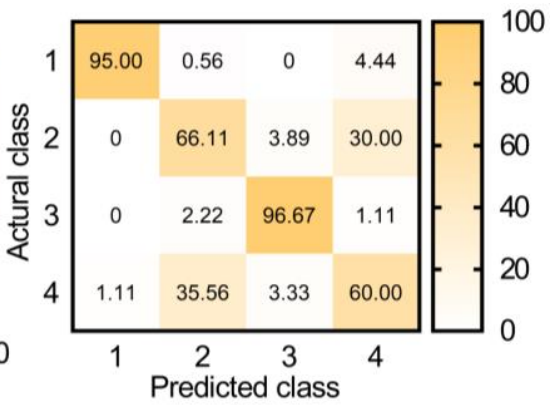

(f)

Fig. 20 Fault diagnosis results of two feature extraction sets using the HHO-SVM: (a) MGRCMSE fitness curve, (b) MGRCMSE output results, (c) MGRCMSE confusion matrix (\%), (d) MGMSE fitness curve, (e) MGMSE output results, and (f) MGMSE confusion matrix (\%) 
(2) Comparison with single-channel feature extraction algorithms. In this section, the MGRCMSE algorithm performance was compared to GMSE, GRCMSE, generalized composite multi-scale fuzzy entropy (GCMFE), and generalized composite multi-scale permutation entropy (GCMPE). These single-channel feature extraction methods were used to extract features from the $\mathrm{X}, \mathrm{Y}$, and $\mathrm{Z}$ channel signals. The extracted results were then submitted to the HHO-SVM classifier for fault identification and the results are shown in Fig. 21. The algorithm parameters used to carry out the study are given in Table 6 .

Table 6 Parameter settings of different feature extraction methods

\begin{tabular}{llll}
\hline Reference & Algorithm & Type & Parameter setting \\
\hline Costa et al. [30] & GMSE & single-channel method & $m=2, r=0.15 s d, N=3000$, and $s=25$ \\
Wang et al. [20] & GRCMSE & single-channel method & $m=2, r=0.15 s d, N=3000$, and $s=25$ \\
Yu et al. [31] & GCMFE & single-channel method & $m=2, r=0.15 s d, n=2, N=3000$, and $s=25$ \\
Zheng et al. [32] & GCMPE & single-channel method & $m=6, \lambda=1, N=3000$, and $s=25$ \\
Wang et al. (proposed) & MGRCMSE & multi-channel method & $m=2, r=0.15 s d, N=3000$, and $s=25$ \\
\hline
\end{tabular}

As can be seen in Fig. 21, the diagnosis accuracy of the same single-channel feature extraction algorithms (i.e., GMSE, GRCMSE, GCMFE, and GCMPE) varied significantly depending on the channel signals. For example, the GRCMSE algorithm had the highest average recognition accuracy for channel $\mathrm{X}$ signals (i.e., $81.25 \%$ ), while it was the lowest for channel Y signals (i.e., 78.19\%). The total difference was 3.06\%. Moreover, the proposed MGRCMSE method had the highest recognition accuracy for bearing fault signals. Thus, it was shown that MGRCMSE can both fully exploit the fusion features of all the channels and explore the fault information more thoroughly compared to existing single-channel feature extraction methods. Lastly, the proposed MGRCMSE algorithm can avoid the channel signal selection effects present in single-channel feature extraction algorithms.

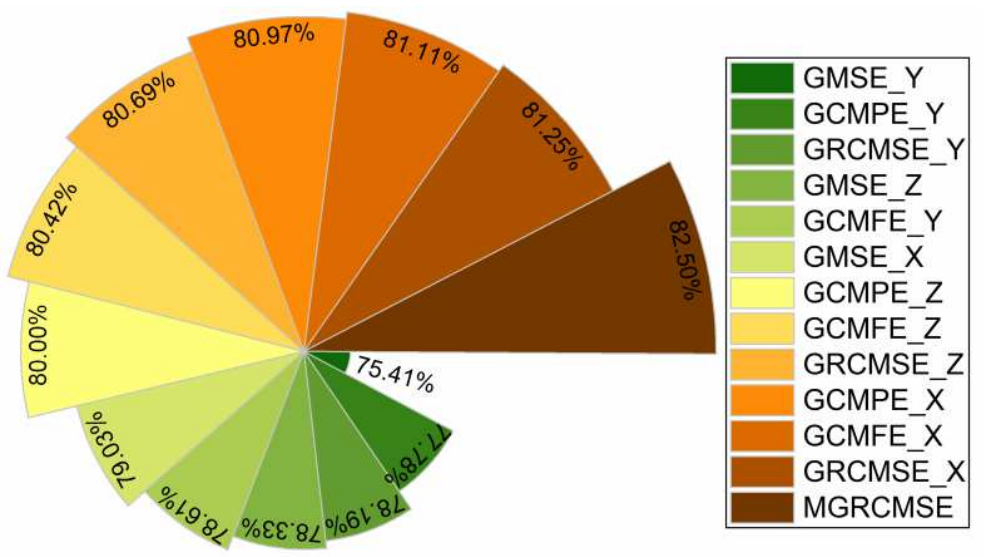

Fig. 21 Fault diagnosis accuracy of various feature extraction algorithms

\section{Conclusions}

In this paper, a novel non-linear dynamics technique, named the multi-variate generalized refined composite multi-scale sample entropy (MGRCMSE), is proposed. The aim was to enhance the complexity of measuring the performance of multi-channel signals. The proposed method was then applied to extract the features of multi-channel rotating machinery signals. Both the simulation analysis and rotating machinery fault diagnosis experiments confirmed the proposed method effectiveness, along with its advantages. The following conclusions were made:

(1) The proposed MGRCMSE algorithm can effectively extract the fusion entropy features for both bearings and gears.

(2) Compared to the existing multi-channel feature extraction algorithm (i.e., MGMSE) and single-channel 
feature extraction algorithms (i.e., GMSE, GRCMSE, GCMFE, GCMPE), the MGRCMSE displayed the highest fault diagnostic accuracy.

(3) The proposed intelligent multi-channel fault diagnosis method, based on MGRMSE, can accurately diagnose both the bearing and gear faults.

This fault diagnosis method presents a research idea for the intelligent fault diagnosis of rotating machinery using multi-channel signals. In the future, the authors aim to apply the proposed method to diagnose similar rotating machinery faults.

\section{Acknowledgments}

This work was supported in part by the National Natural Science Foundation of China (Grant No. 51775114, 51875105, 51275092); the Fujian Provincial Industrial Robot Basic Components Technology Research and Development Center (Grant No. 2014H21010011); and the Natural Science Foundation of Anhui Province (Grant No. 1808085ME152).

\section{Compliance with ethical standards}

\section{Conflict of interest}

The authors declare that they have no conflict of interest.

\section{References}

[1] Prabith, K., Praveen Krishna, I. R.: The numerical modeling of rotor-stator rubbing in rotating machinery: A comprehensive review. Nonlinear Dynamic 101, 1317-1363 (2020)

https://doi.org/10.1007/s11071-020-05832-y

[2] Zheng, J., Pan., H.: Use of generalized refined composite multiscale fractional dispersion entropy to diagnose the faults of rolling bearing. Nonlinear Dynamic 101, 1417-1440 (2020) https://doi.org/10.1007/s11071-020-05821-1

[3] Li, X., Li, J., Zhao, C., Qu, Y., He, D.: Gear pitting fault diagnosis with mixed operating conditions based on adaptive 1D separable convolution with residual connection. Mechanical Systems and Signal Processing 142, 106740 (2020) https://doi.org/10.1016/j.ymssp.2020.106740

[4] Pang, S., Yang, X., Zhang, X., Lin, X.: Fault diagnosis of rotating machinery with ensemble kernel extreme learning machine based on fused multi-domain features. ISA Transactions 98, 320-337 (2020) https://doi.org/10.1016/j.isatra.2019.08.053

[5] Lei, Y., Yang, B., Jia, F., Li, N., Nandi, A. K.: Applications of machine learning to machine fault diagnosis: A review and roadmap. Mechanical Systems and Signal Processing 138, 106587 (2020) https://doi.org/10.1016/j.ymssp.2019.106587

[6] Xue, Y., Dou, D., Yang, J.: Multi-fault diagnosis of rotating machinery based on deep convolution neural network and support vector machine. Measurement 156, 107571 (2020) https://doi.org/10.1016/j.measurement.2020.107571

[7] Zhao, B., Zhang, X., Li, H., Yang, Z.: Intelligent fault diagnosis of rolling bearings based on normalized CNN considering data imbalance and variable working conditions. Knowledge-Based Systems 199, 105971 (2020) https://doi.org/10.1016/j.knosys.2020.105971 
[8] Zhang, Z., Li, S., Lu, J., Wang, J., Jiang, X.: A novel intelligent fault diagnosis method based on fast intrinsic component filtering and pseudo-normalization. Mechanical Systems and Signal Processing 145, 106923 (2020) https://doi.org/10.1016/j.ymssp.2020.106923

[9] Li, Y., Wang, S., Deng, Z.: Intelligent fault identification of rotary machinery using refined composite multi-scale Lempel-Ziv complexity. Journal of Manufacturing Systems (2020) (in Press) https://doi.org/10.1016/j.jmsy.2020.05.004

[10] Wu, Y., Jiang, P., Ding, C., Feng, F., Chen, T.: Intelligent fault diagnosis of rotating machinery based on one-dimensional convolutional neural network. Computers in Industry 108, 53-61 (2019) https://doi.org/10.1016/j.compind.2018.12.001

[11] Zhao, X., Jia, M., Ding, P., Yang, C., She, D., Liu, Z.: Intelligent fault diagnosis of multi-channel motor-rotor system based on multi-manifold deep extreme learning machine. IEEE/ASME Transactions on Mechatronics 25(5), 2177-2187 (2020) https://doi.org/10.1109/TMECH.2020.3004589

[12] Shao, S., Yan, R., Lu, Y., Wang, P., Gao, R. X.: DCNN-based multi-signal induction motor fault diagnosis. IEEE Transactions on Instrumentation and Measurement 69(6), 2658-2669 (2020) https://doi.org/10.1109/TIM.2019.2925247

[13] Wu, J., Jiang, B., Chen, H., Liu, J.: Sensors information fusion system with fault detection based on multi-manifold regularization neighborhood preserving embedding. Sensors 19(6), 1440 (2019) https://doi.org/10.3390/s19061440

[14] Wang, Z., Yao, L., Cai, Y., Zhang, J.: Mahalanobis semi-supervised mapping and beetle antennae search based support vector machine for wind turbine rolling bearings fault diagnosis. Renewable Energy 155, 1312-1327 (2020) https://doi.org/10.1016/j.renene.2020.04.041

[15] Teng, Y., Shang, P., He, J.: Multiscale fractional-order approximate entropy analysis of financial time series based on the cumulative distribution matrix. Nonlinear Dynamics 97, 1067-1085 (2019) https://doi.org/10.1007/s11071-019-05033-2

[16] Liu, H., Han, M.: A fault diagnosis method based on local mean decomposition and multi-scale entropy for roller bearings. Mechanism and Machine Theory 75, 67-78 (2014) https://doi.org/10.1016/j.mechmachtheory.2014.01.011

[17] Landauskas, M., Cao, M., Ragulskis, M.: Permutation entropy-based 2D feature extraction for bearing fault diagnosis. Nonlinear Dynamics 102, 1717-1731 (2020) https://doi.org/10.1007/s11071-020-06014-6

[18] Zheng, J., Jiang, Z., Pan, H.: Sigmoid-based refined composite multi-scale fuzzy entropy and t-SNE based fault diagnosis approach for rolling bearing. Measurement 129, 332-342 (2018) https://doi.org/10.1016/j.measurement.2018.07.045

[19] Yan, X., Jia, M.: Intelligent fault diagnosis of rotating machinery using improved multiscale dispersion entropy and mRMR feature selection. Knowledge-Based Systems 163, 450-471 (2019) https://doi.org/10.1016/j.knosys.2018.09.004

[20] Wang, Z., Yao, L., Cai, Y.: Rolling bearing fault diagnosis using generalized refined composite multi-scale 
sample entropy and optimized support vector machine. Measurement 156, 107574 (2020)

https://doi.org/10.1016/j.measurement.2020.107574

[21] Wang, C.: A sample entropy inspired affinity propagation method for bearing fault signal classification. Digital Signal Processing 102, 102740 (2020)

https://doi.org/10.1016/j.dsp.2020.102740

[22] Gao, Q., Liu, W., Tang, B., Li, G.: A novel wind turbine fault diagnosis method based on intergral extension load mean decomposition multi-scale entropy and least squares support vector machine. Renewable Energy 116, 169-175 (2018) https://doi.org/10.1016/j.renene.2017.09.061

[23] Dai, J., Zheng, J., Pan, H., Pan, Z.: Rolling bearing fault diagnosis method based on composite multi-scale entropy and Laplacian SVM. China Mechanical Engineering 28(11), 1339-1346 (2017) https://doi.org/10.3969/j.issn.1004-132X.2017.11.014

[24] Lu, Y., Wang, J.: Multi-variate multi-scale entropy of financial markets. Communications in Nonlinear Science and Numerical Simulation 52, 77-90 (2017) https://doi.org/10.1016/j.cnsns.2017.04.028

[25] Humeau-Heurtier, A.: Multi-variate refined composite multi-scale entropy analysis. Physics Letters A 380(16), 1426-1431 (2016) https://doi.org/10.1016/j.physleta.2016.02.029

[26] Yin, Y., Wang, X., Li, Q., Shang, P.: Generalized multi-variate multi-scale sample entropy for detecting the complexity in complex systems. Physica A: Statistical Mechanics and Its Applications 545, 123814 (2020) https://doi.org/10.1016/j.physa.2019.123814

[27] Houssein, E. H., Hosney, M. E., Oliva, D., Mohamed, W. M., Hassaballah, M.: A novel hybrid Harris hawks optimization and support vector machines for drug design and discovery. Computers and Chemical Engineering 133, $106656(2020)$ https://doi.org/10.1016/j.compchemeng.2019.106656

[28] Machado, J. T., Lopes, A. M.: Multidimensional scaling and visualization of patterns in prime numbers. Communications in Nonlinear Science and Numerical Simulation 83, 105128 (2020) https://doi.org/10.1016/j.cnsns.2019.105128

[29] Heidari, A. A., Mirjalili, S., Faris, H., Aljarah, I., Mafarja, M., Chen, H.: Harris hawks optimization: Algorithm and applications. Future Generation Computer Systems 97, 849-872 (2019) https://doi.org/10.1016/j.future.2019.02.028

[30] Costa1, M. D., Goldberger, A. L.: Generalized multiscale entropy analysis: Application to quantifying the complex volatility of human heartbeat time series. Entropy 17(3), 1197-1203 (2015) https://doi.org/10.3390/e17031197

[31] Wei, Y., Li, Y., Xu, M., Huang, W.: Intelligent fault diagnosis of rotating machinery using ICD and generalized composite multi-scale fuzzy entropy. IEEE Access 7, 38983-38995 (2019) https://doi.org/10.1109/ACCESS.2018.2876759

[32] Zheng, J., Pan, H., Yang, S., Cheng, J.: Generalized composite multiscale permutation entropy and Laplacian score based rolling bearing fault diagnosis. Mechanical Systems and Signal Processing 99, 229-243 (2018) https://doi.org/10.1016/j.ymssp.2017.06.011 


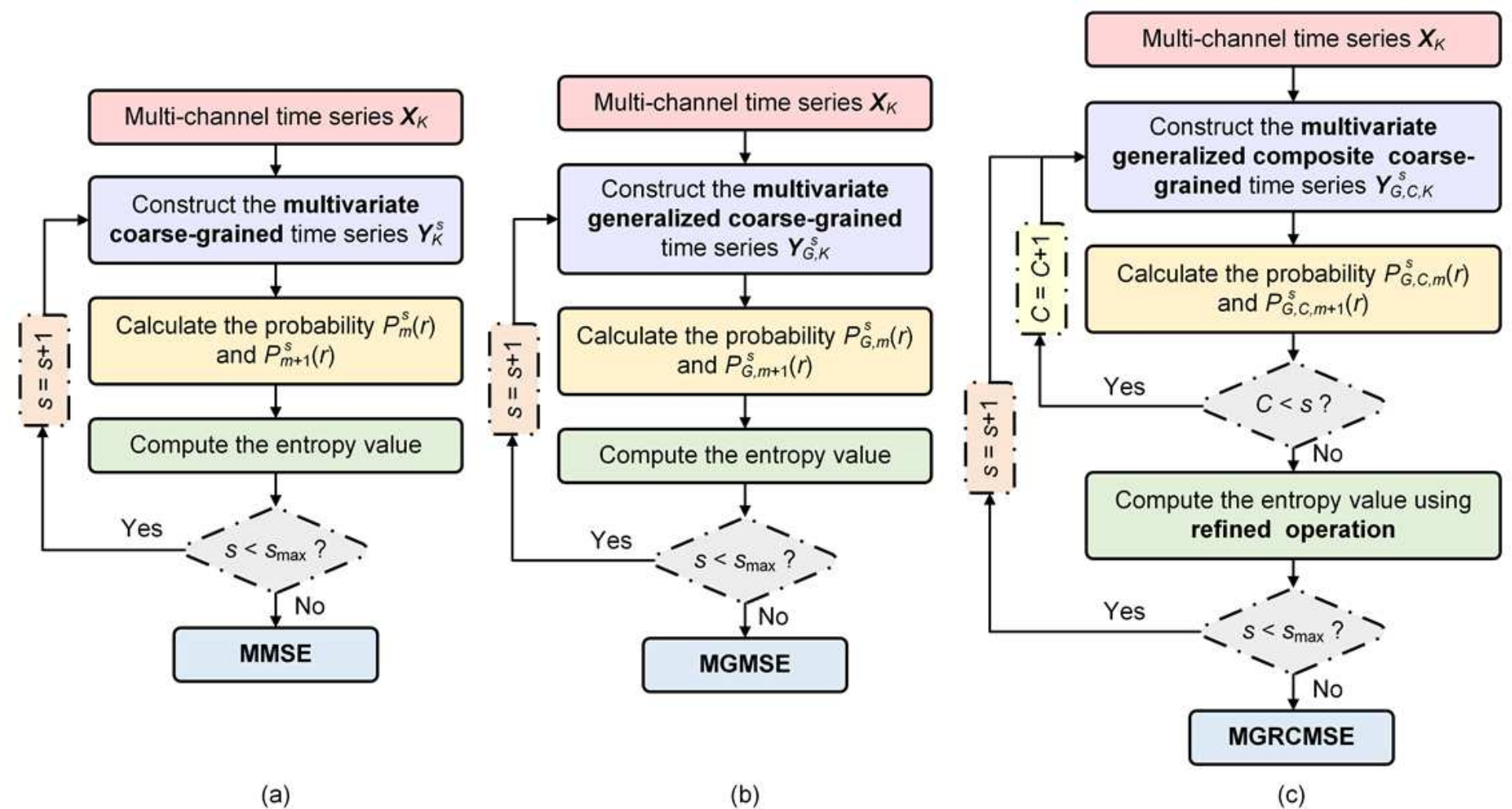

\section{Figure 1}

Flowchart of three multi-channel feature extraction algorithms: (a) MMSE, (b) MGMSE, and (c) MGRCMSE 


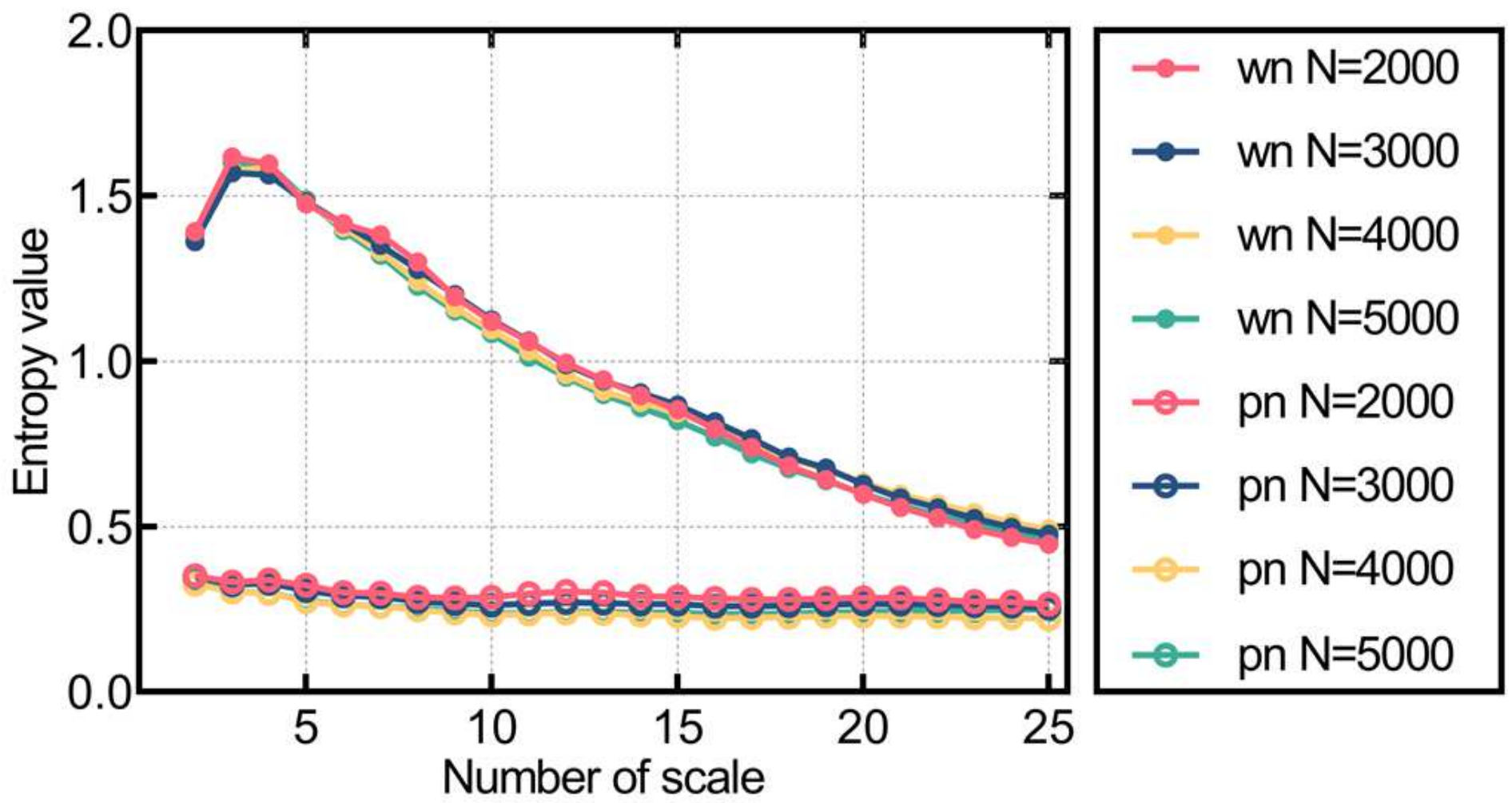

Figure 2

Influence of N on MGRCMSE 


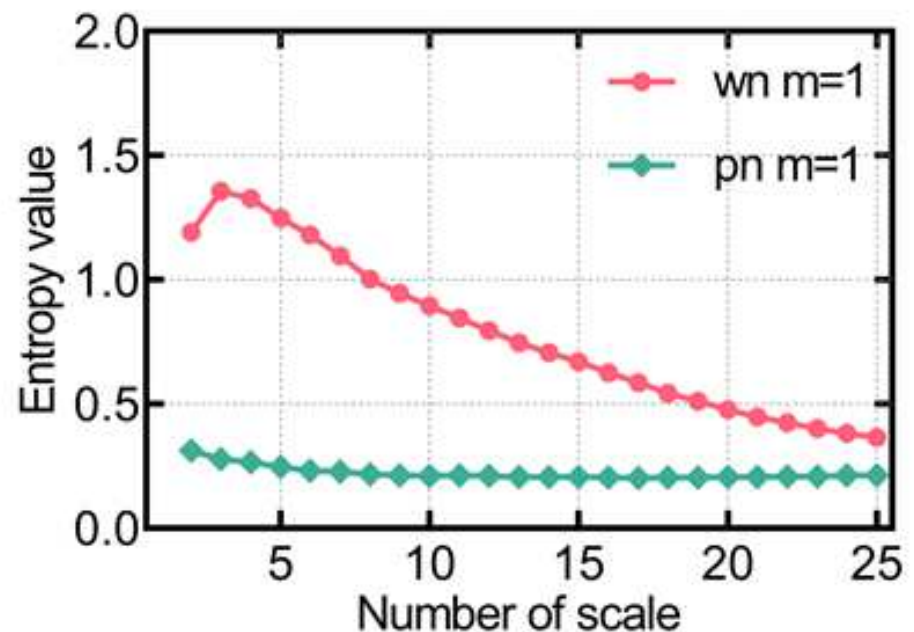

(a)

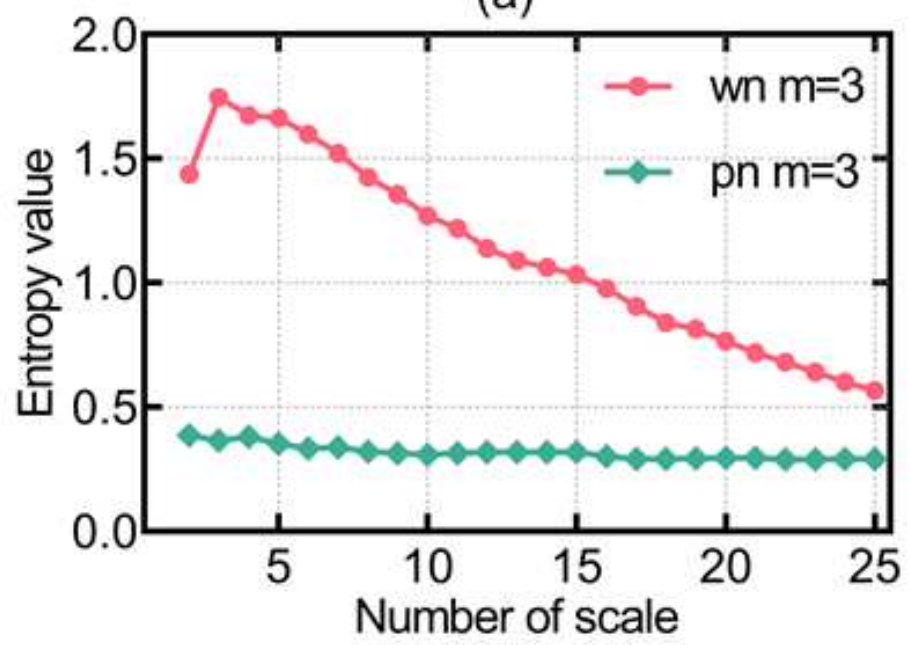

(c)

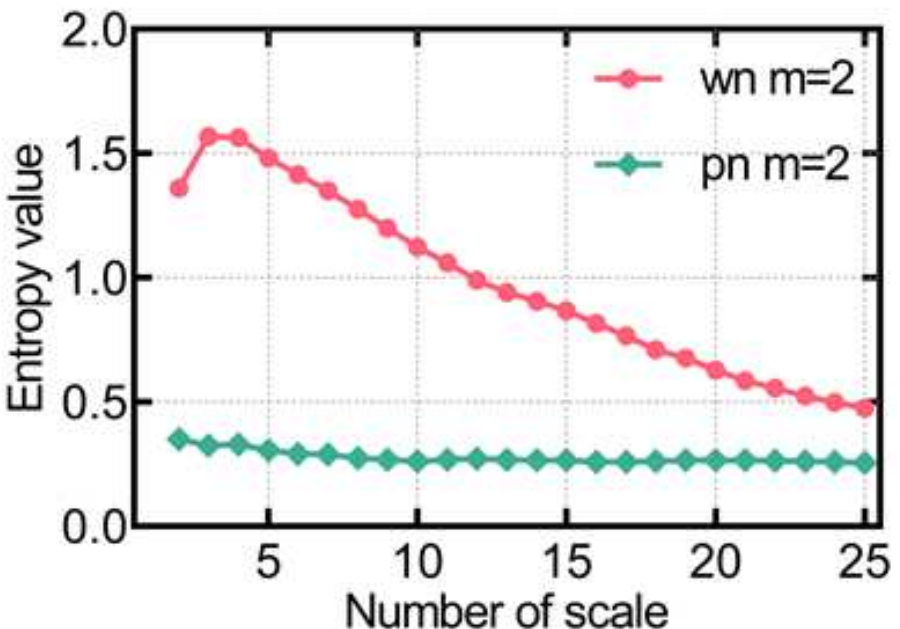

(b)

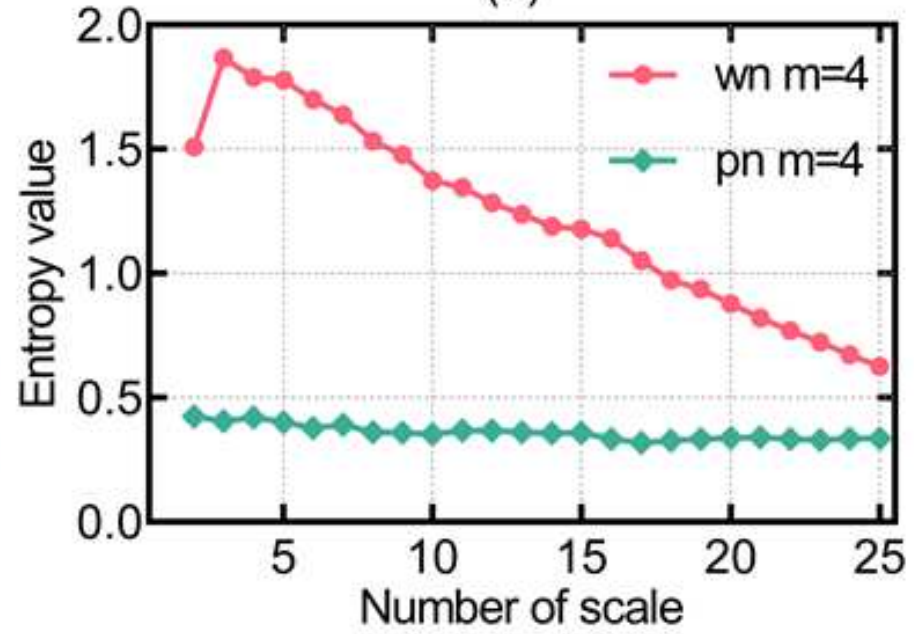

(d)

Figure 3

Influence of $m$ on MGRCMSE: (a) $m=1$, (b) $m=2$, (c) $m=3$, and (d) $m=4$ 


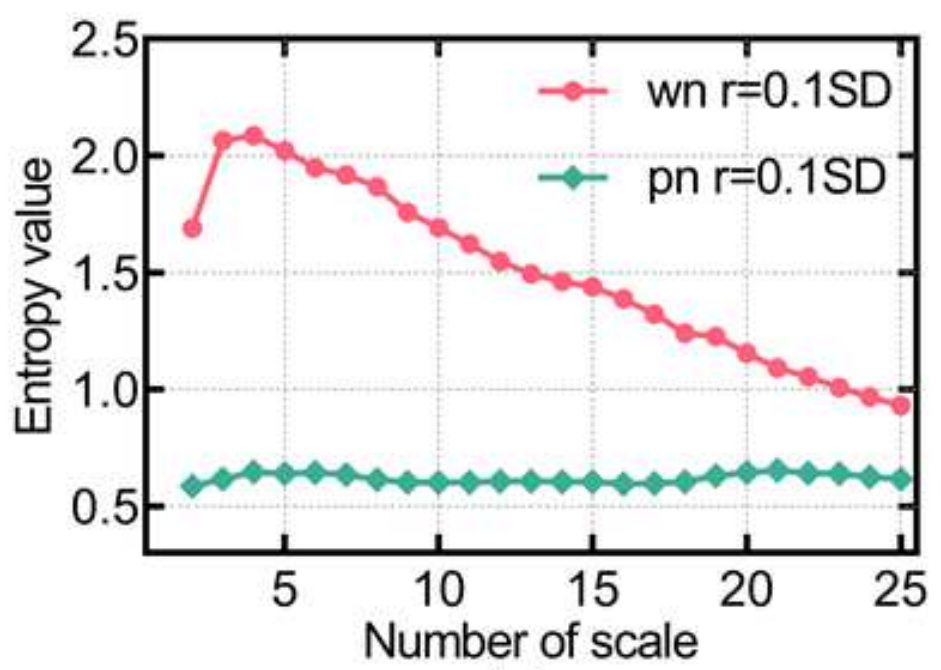

(a)

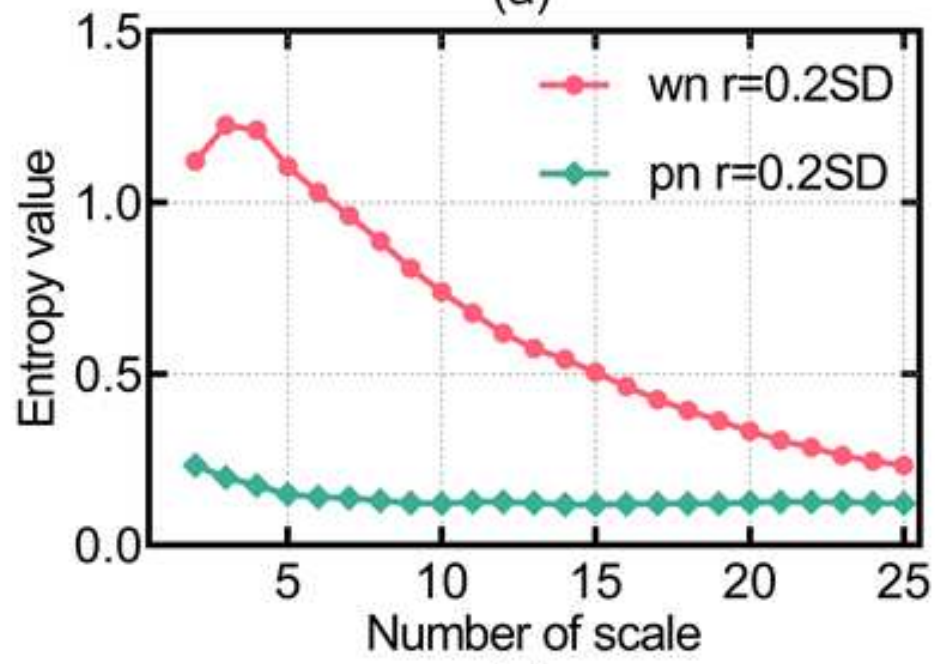

(c)

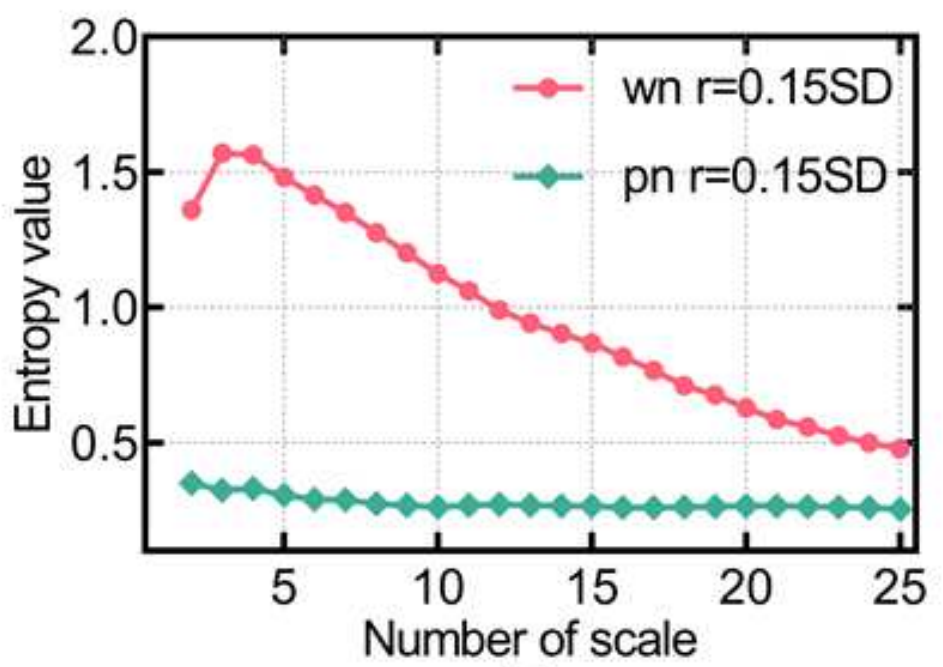

(b)

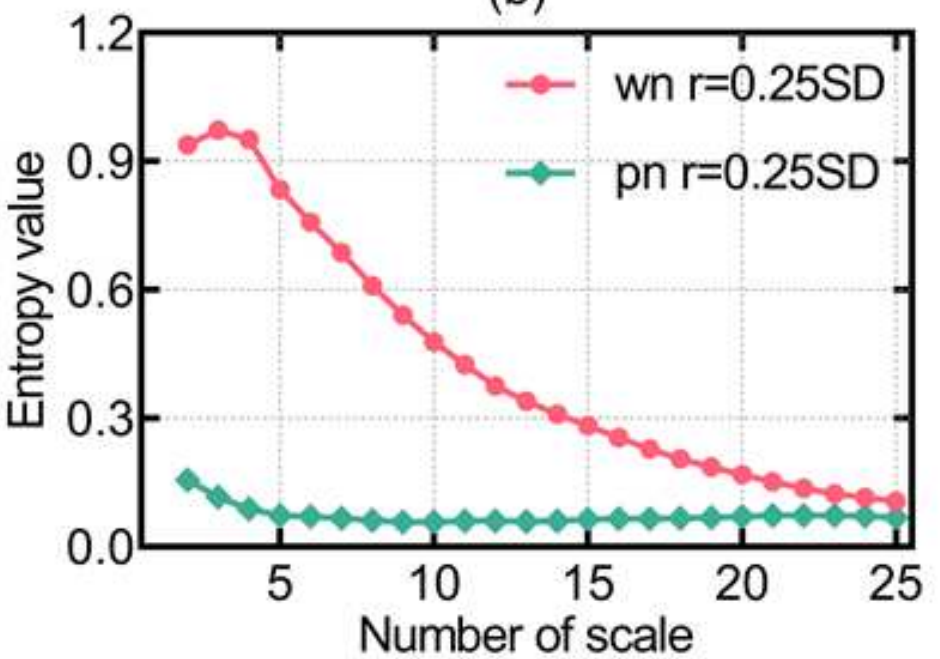

(d)

Figure 4

Influence of $r$ on MGRCMSE: (a) $r=0.1 s d$, (b) $r=0.15 s d$, (c) $r=0.2 s d$, and (d) $r=0.25 s d$ 


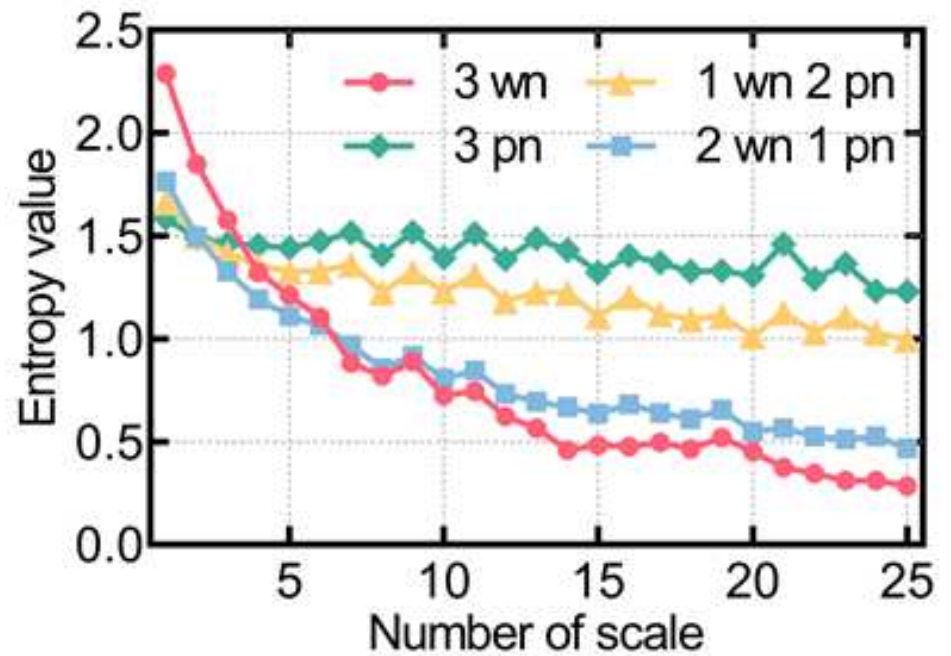

(a)

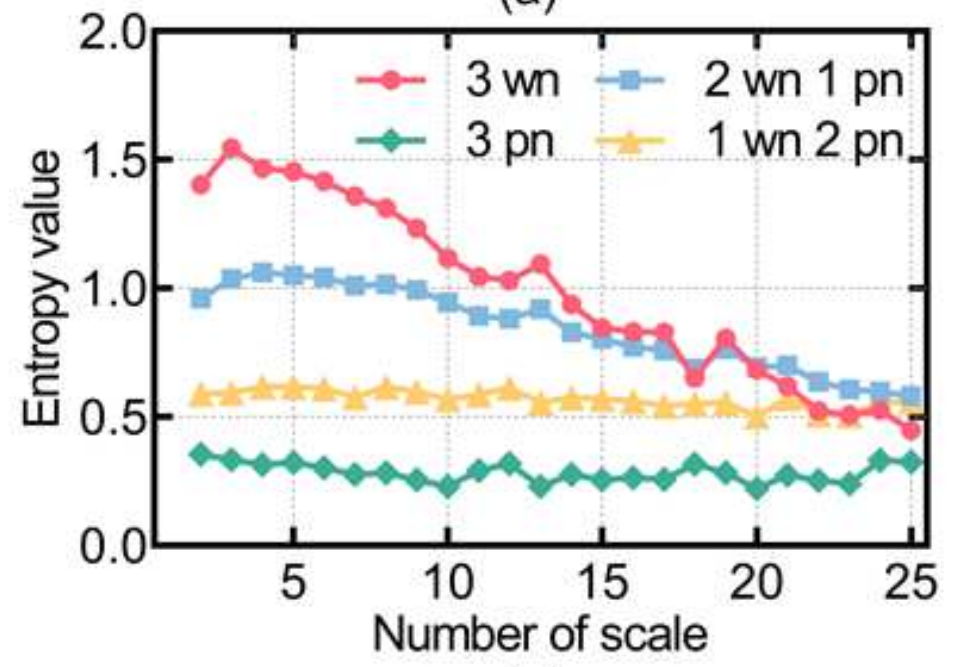

(c)

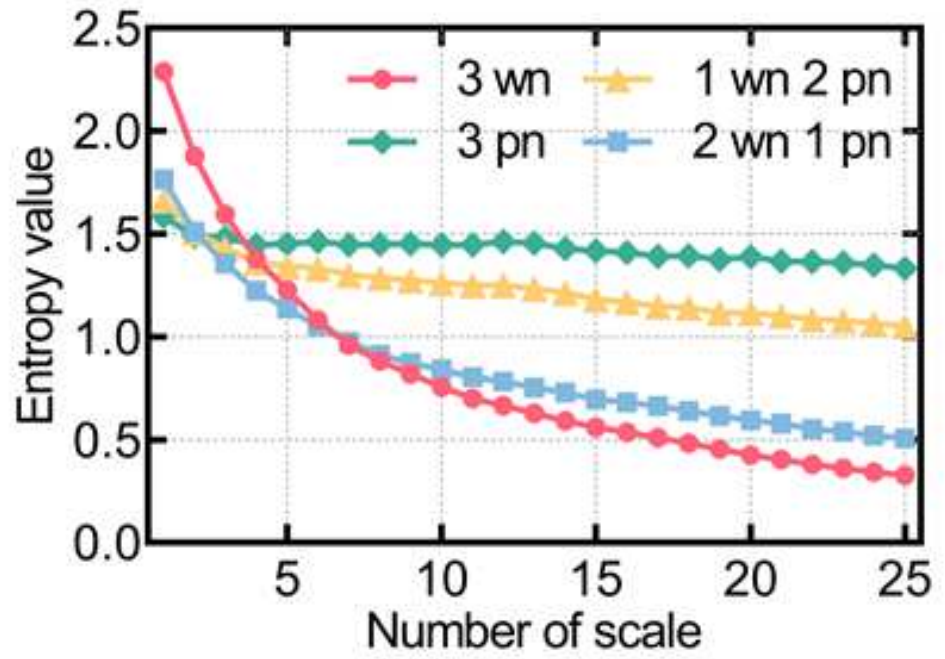

(b)

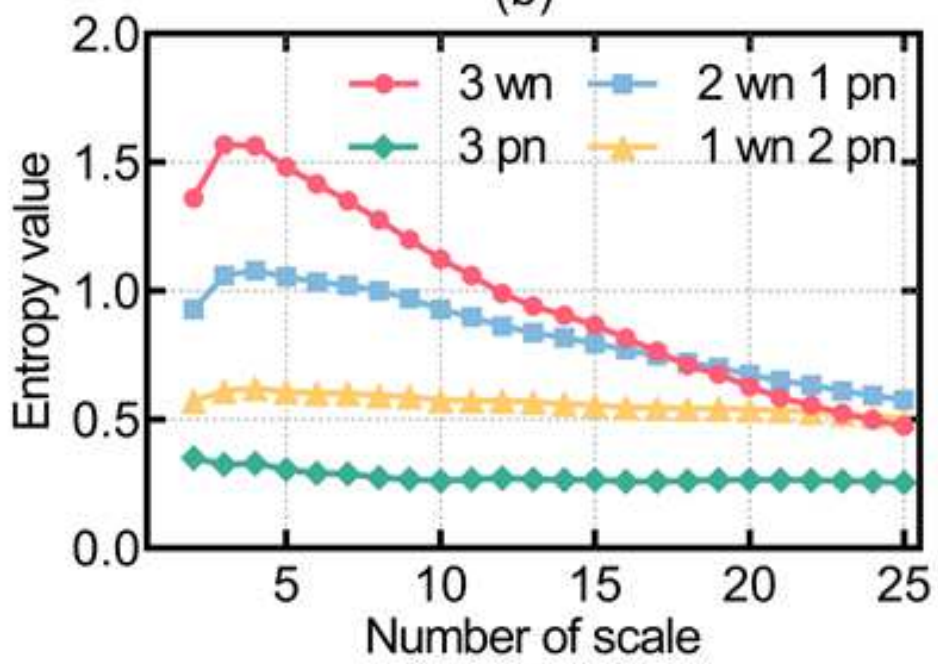

(d)

Figure 5

Analysis results of four multi-channel signal analysis algorithms: (a) MMSE, (b) MRCMSE, (c) MGMSE, and (d) MGRCMSE 

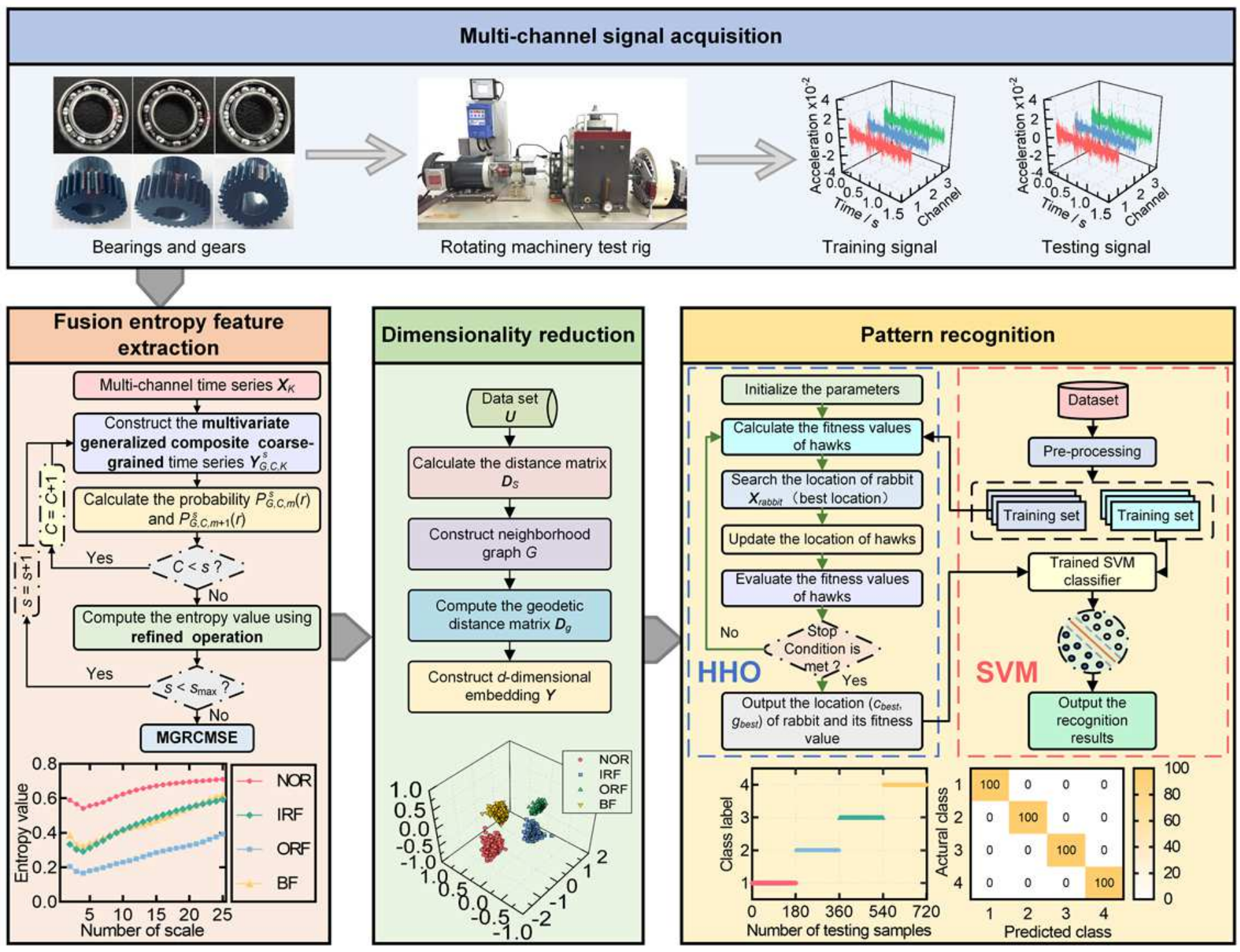

\section{Figure 6}

Flowchart of the proposed intelligent multi-channel fault diagnosis model 


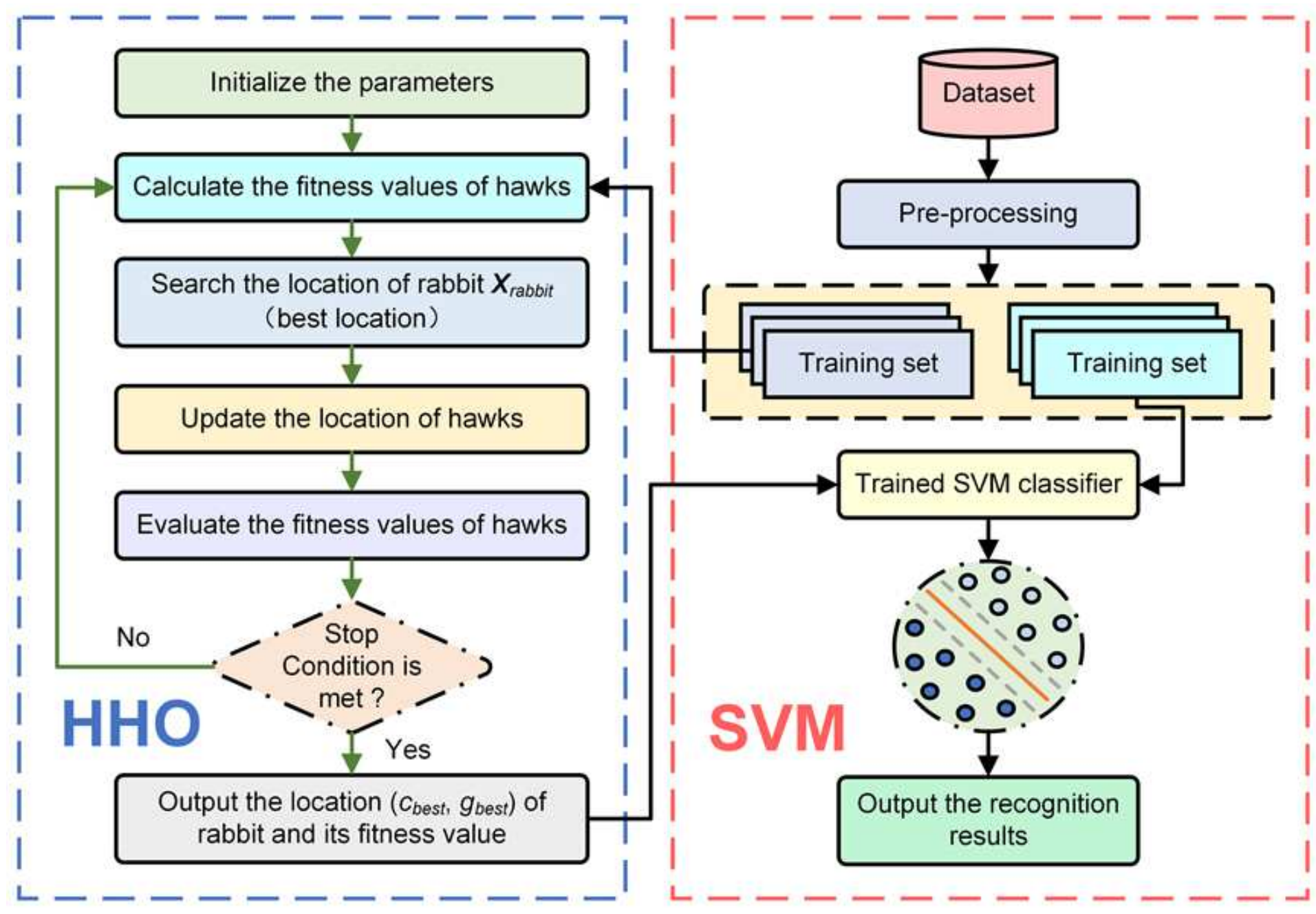

Figure 7

The HHO-SVM flowchart 


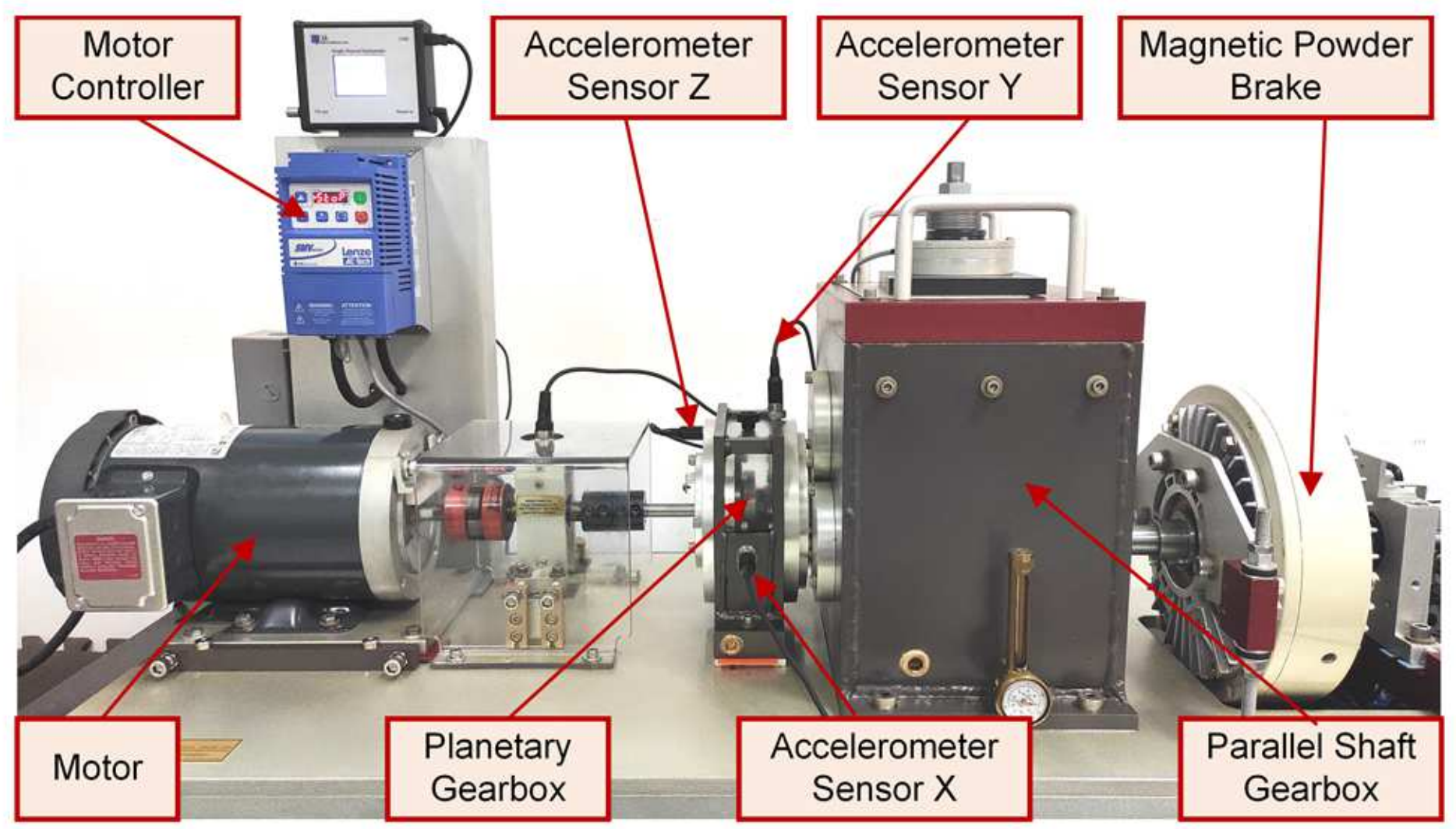

Figure 8

Experimental test platform

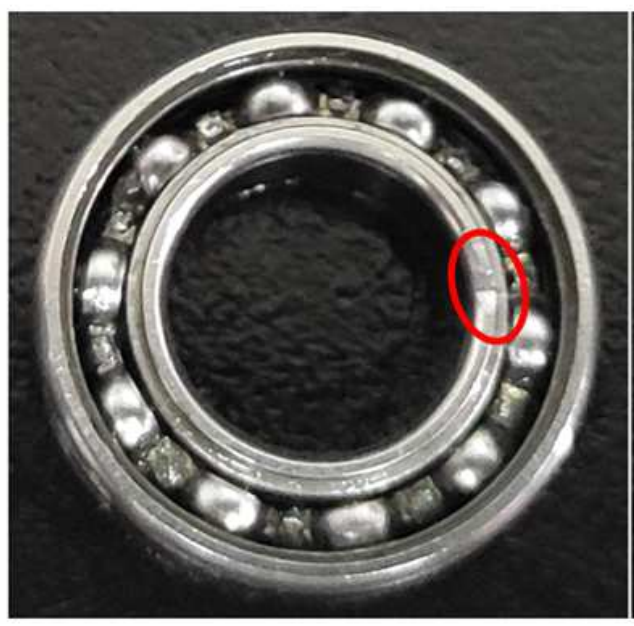

(a)

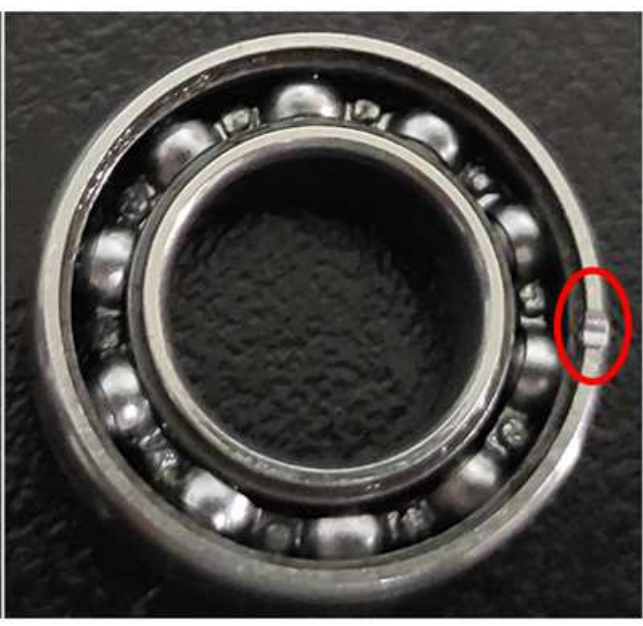

(b)

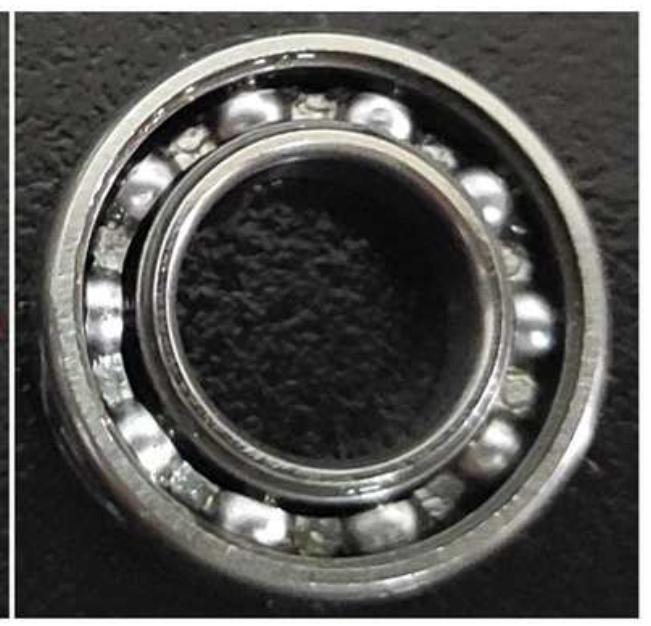

(c)

Figure 9

Bearing faults: (a) inner ring fault, (b) outer ring fault, and (c) ball fault 


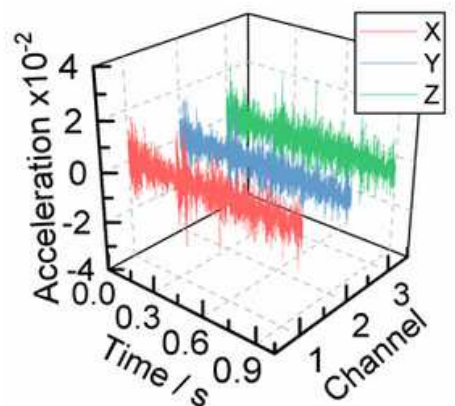

(a)

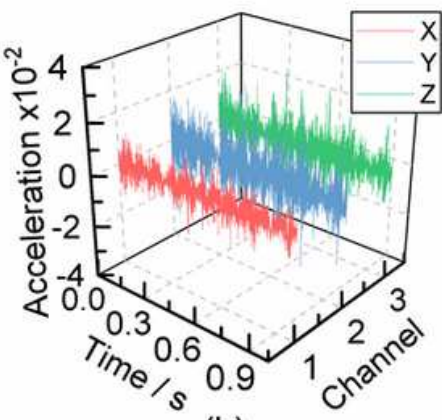

(b)

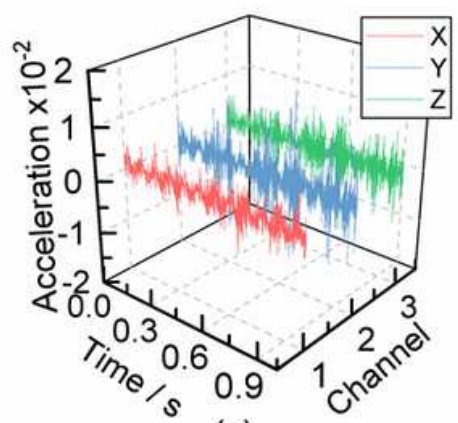

(c)

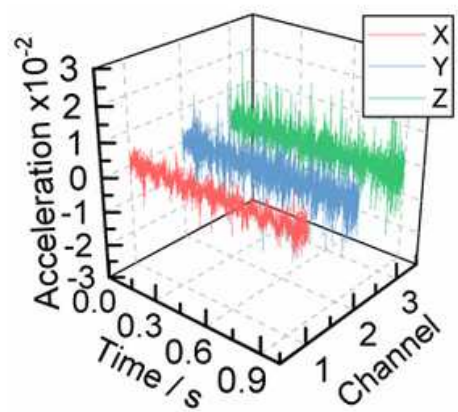

(d)

Figure 10

Time-domain waveforms of rolling bearing vibration signals: (a) NOR, (b) IRF, (c) ORF, and (d) BF
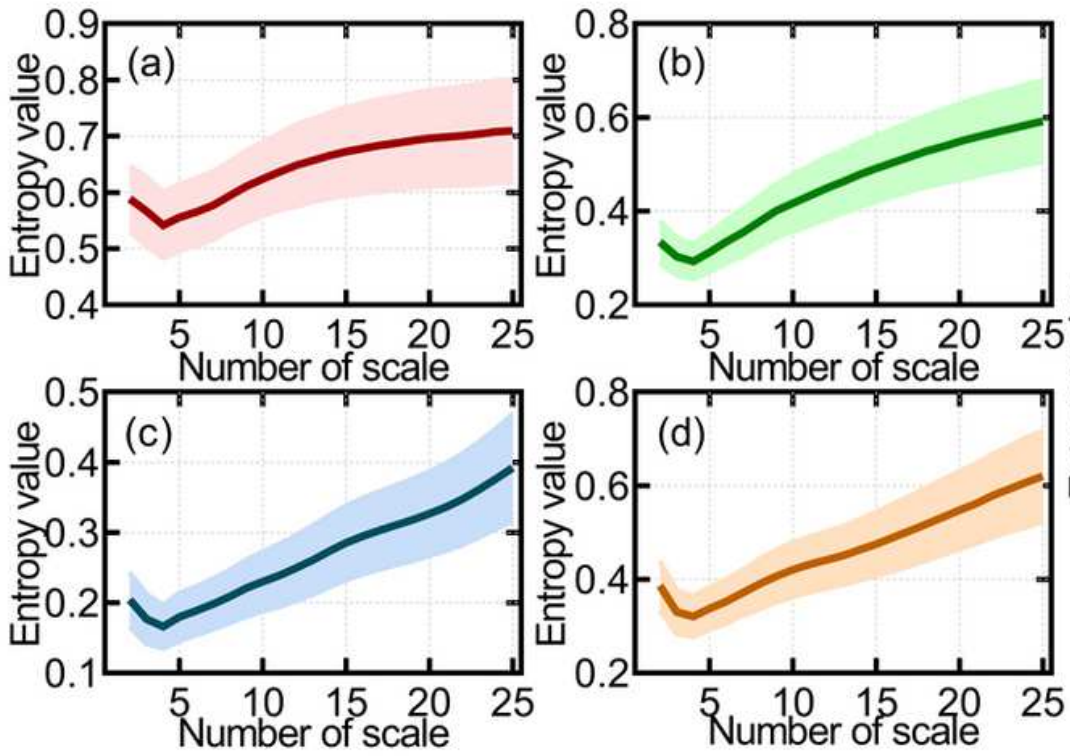

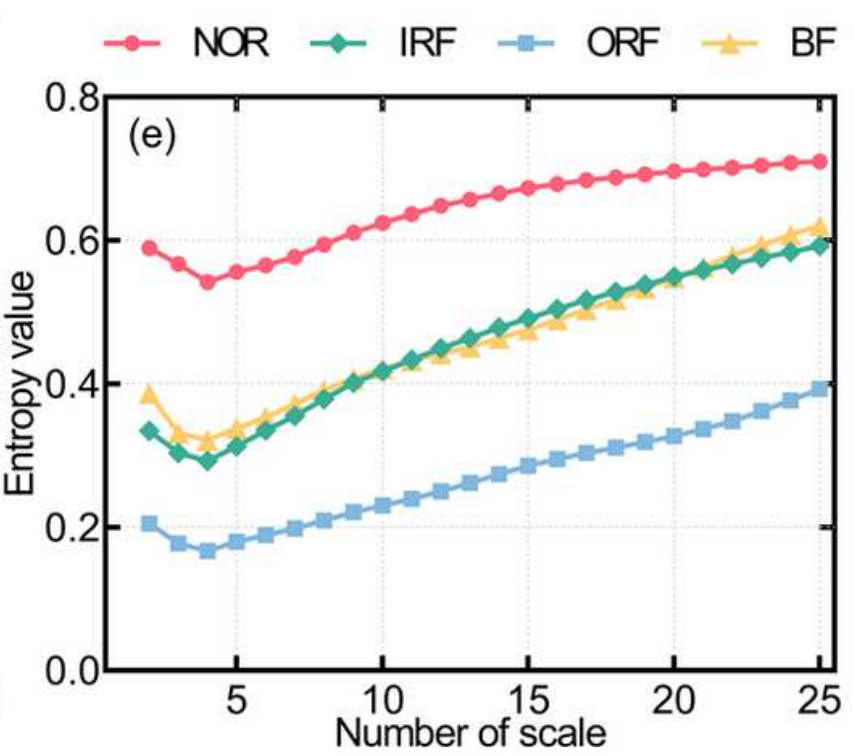

Figure 11

The MGRCMSE entropy curves: (a) NOR, (b) IRF, (c) ORF, (d) BF, and (e) mean MGRCMSE curves 


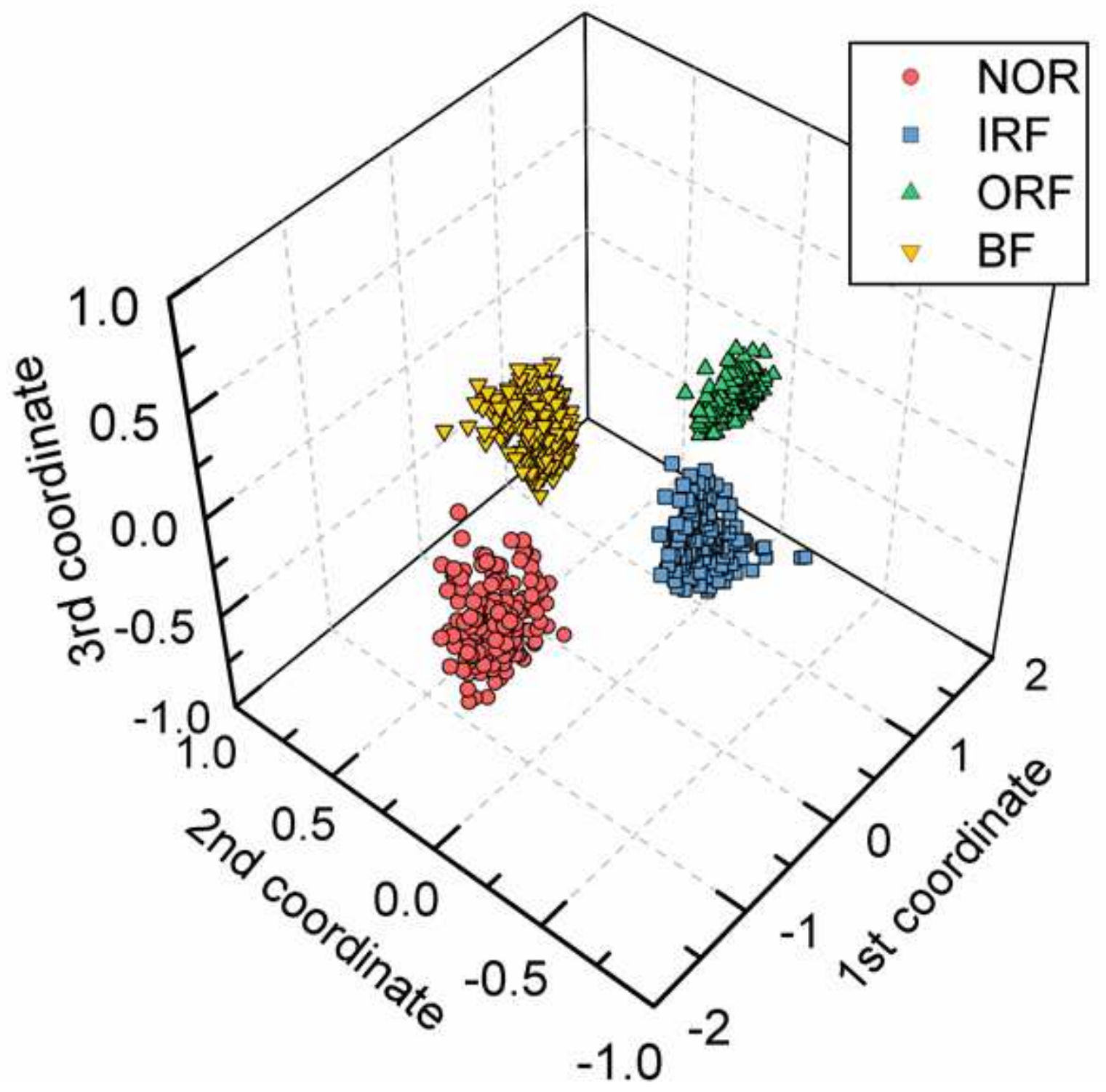

Figure 12

The 3D result obtained using the SIM

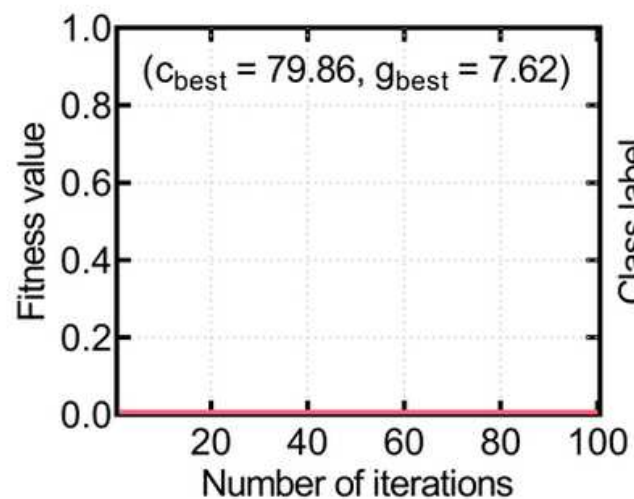

(a)

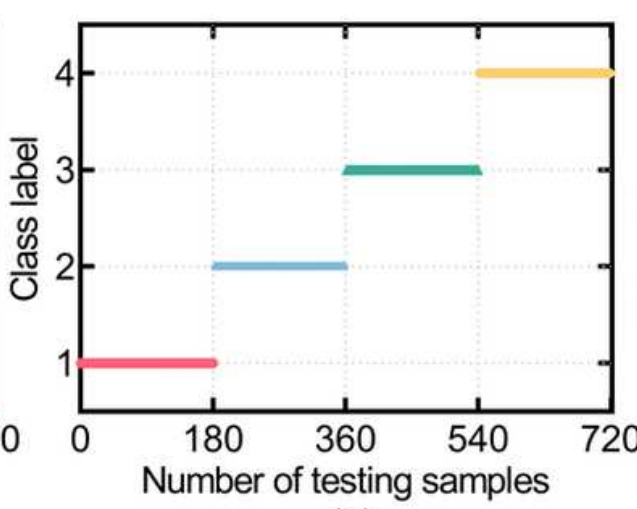

(b)

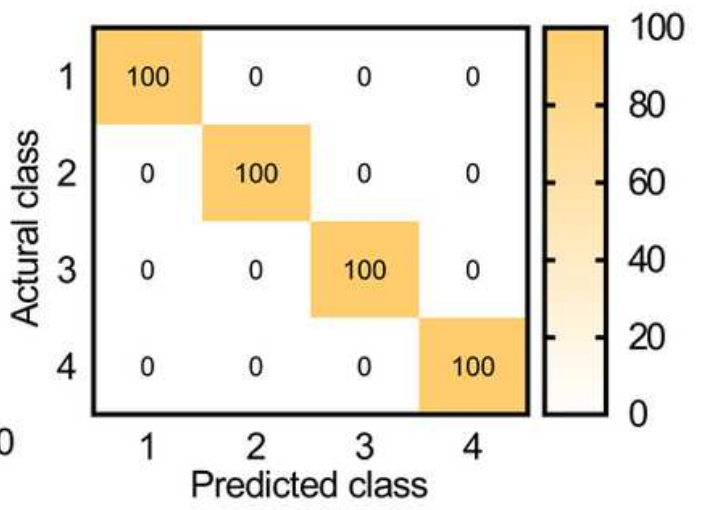

(c)

Figure 13 
Fault diagnosis results: (a) fitness curve, (b) output results, and (c) confusion matrix (\%)

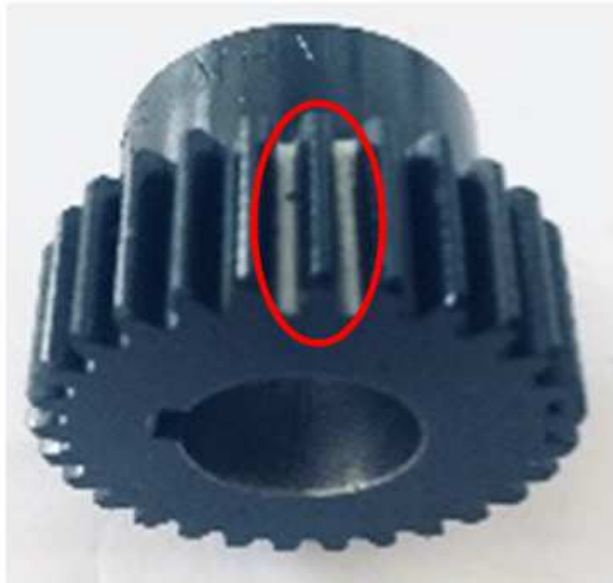

(a)

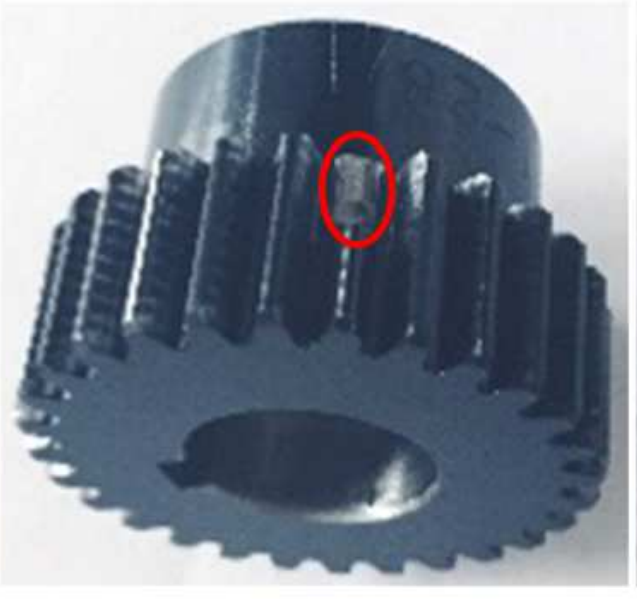

(b)

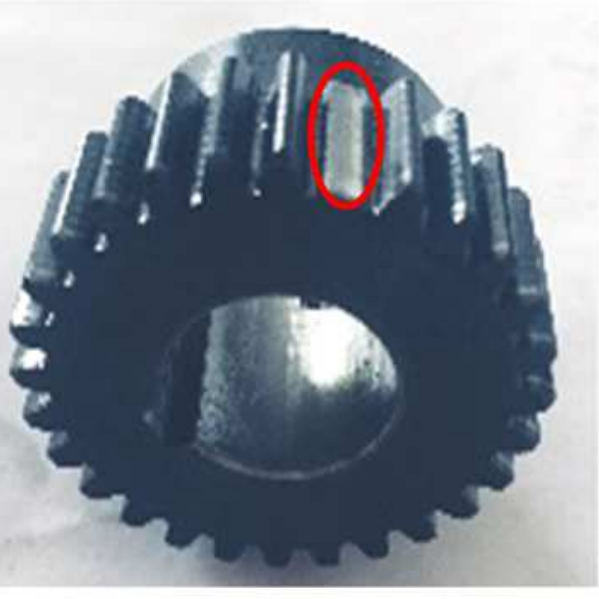

(c)

\section{Figure 14}

Gear faults: (a) tooth root fault, (b) tooth breakage fault, and (c) missing tooth fault

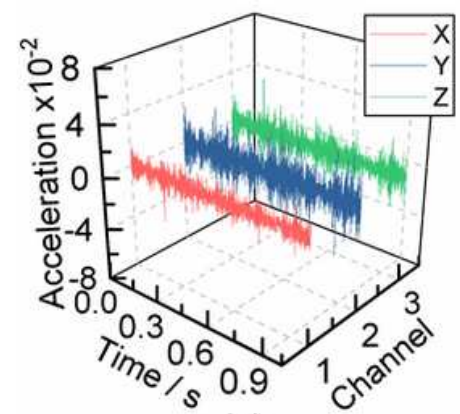

(a)

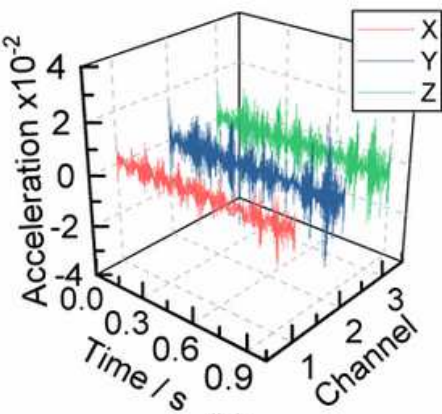

(b)

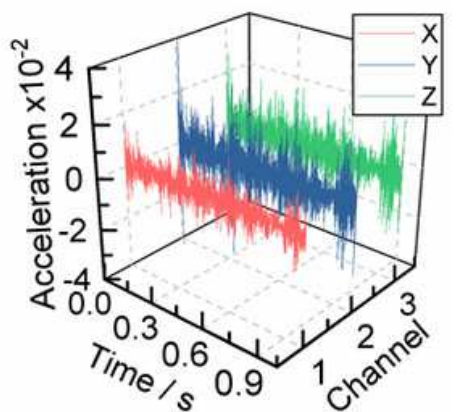

(c)

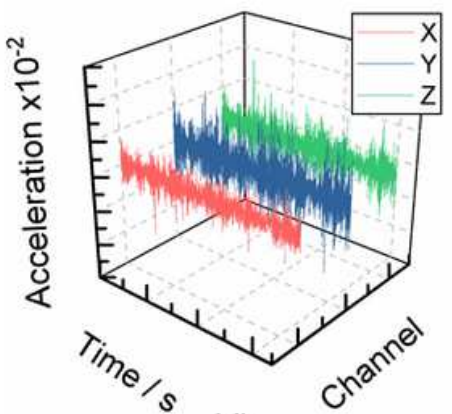

(d)

\section{Figure 15}

Time-domain waveforms of three-channel gear vibration signals: (a) NOR, (b) TBF, (c) MTF, and (d) TRF 


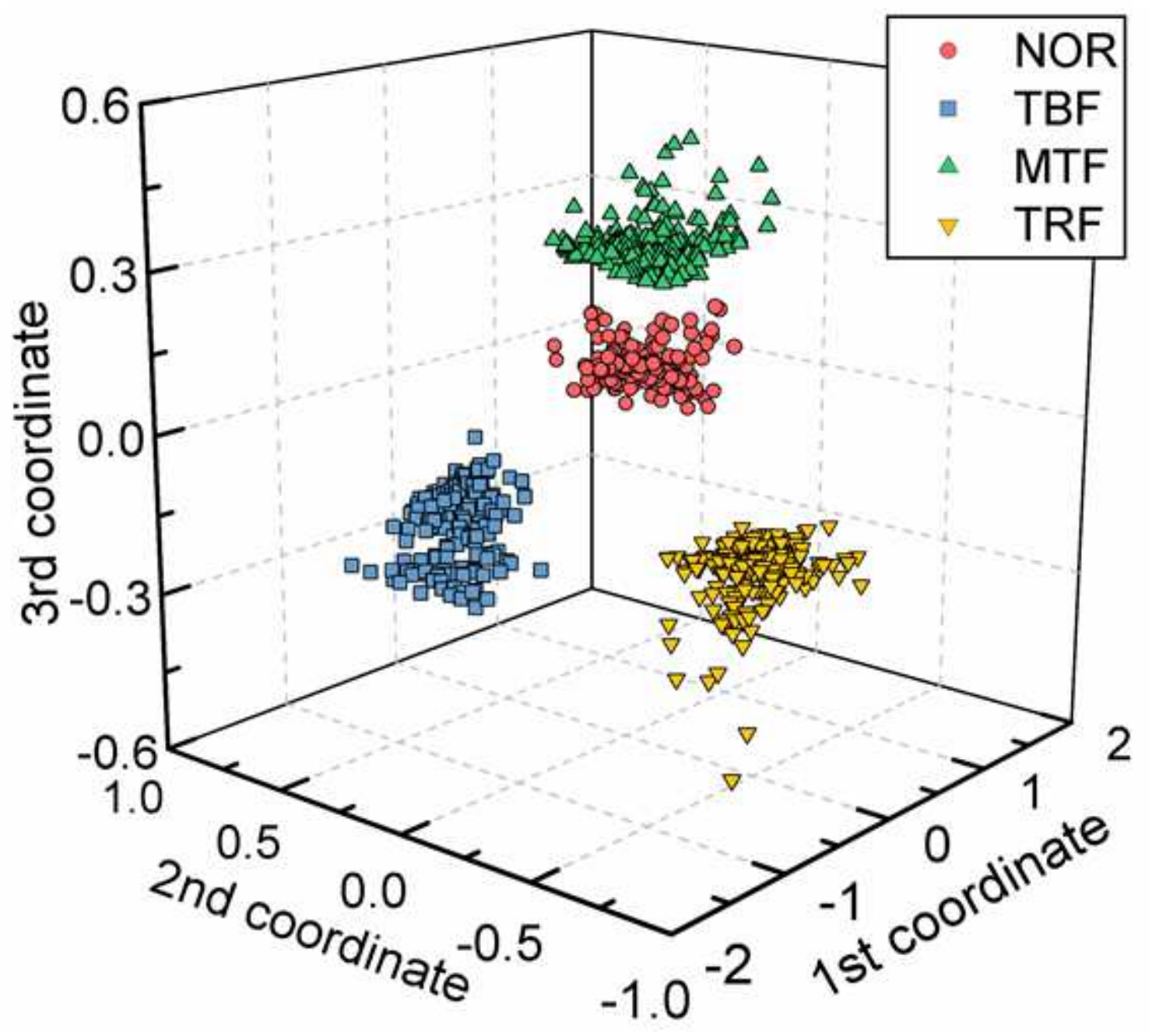

Figure 17

The 3D result obtained using the SIM

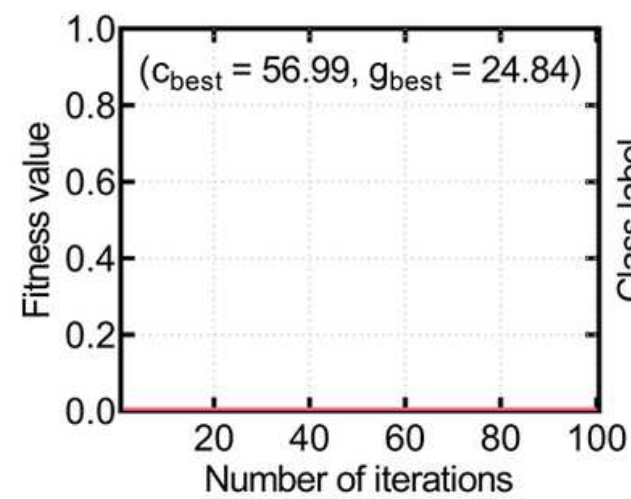

(a)

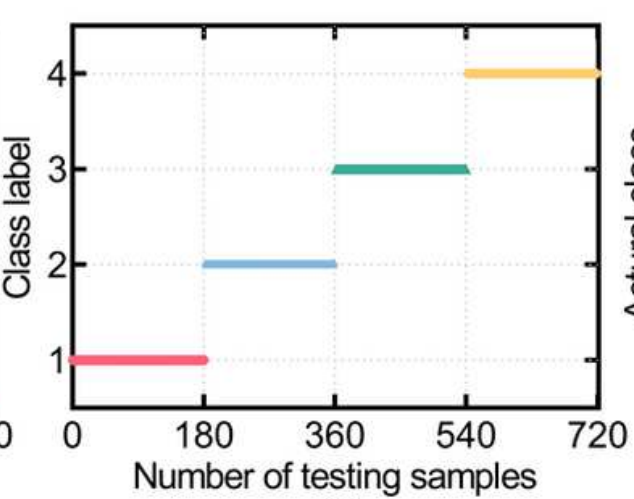

(b)

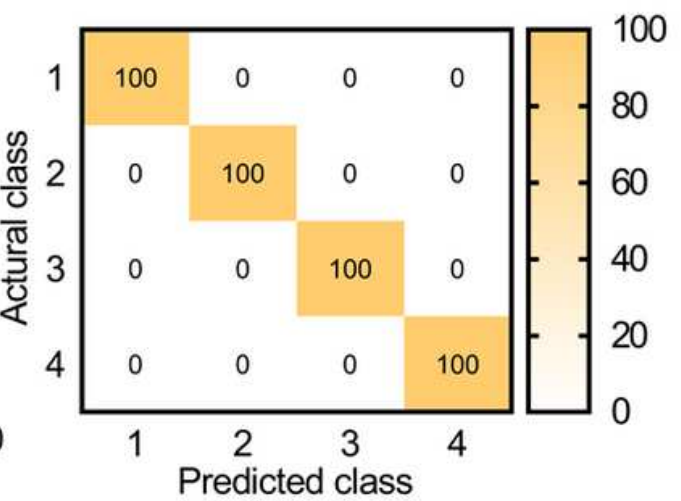

(c)

Figure 18

Fault diagnosis results: (a) fitness curve, (b) output result, and (c) confusion matrix (\%) 


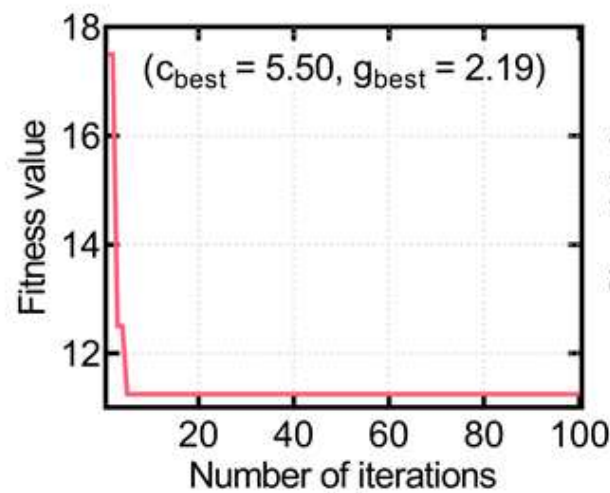

(a)

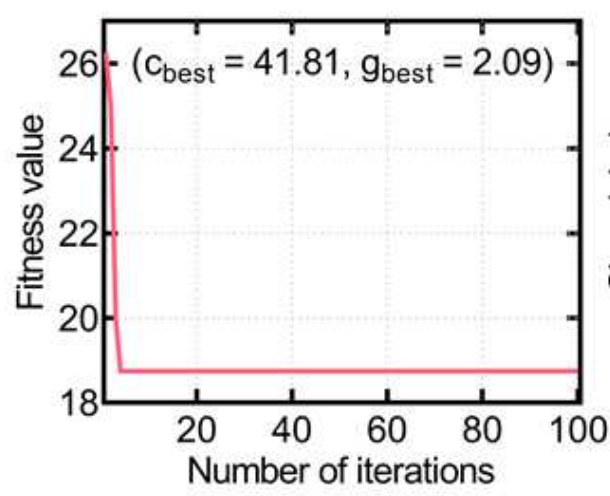

(d)

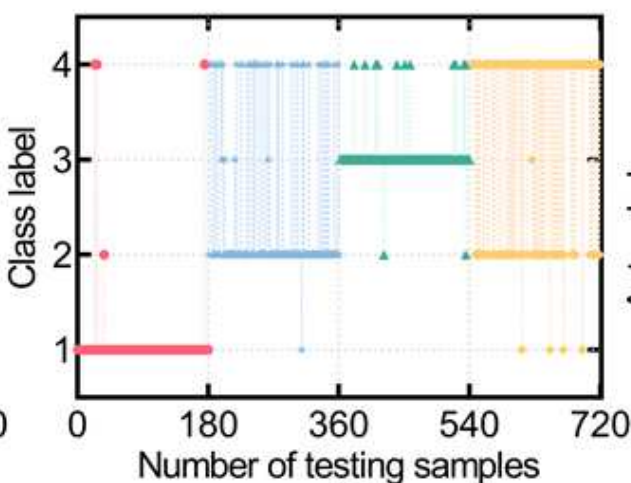

(b)

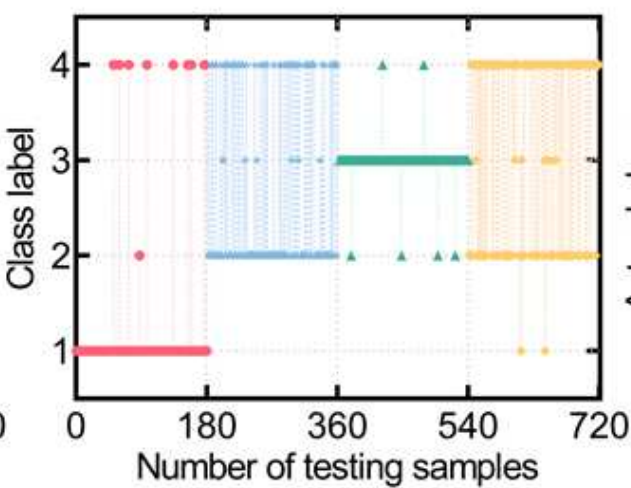

(e)

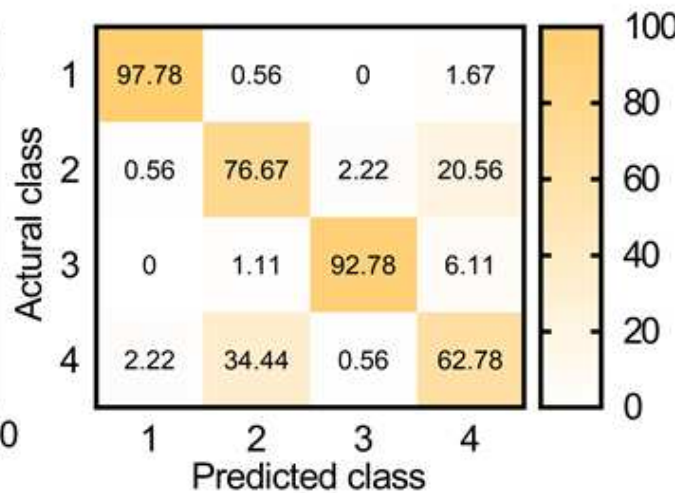

(c)

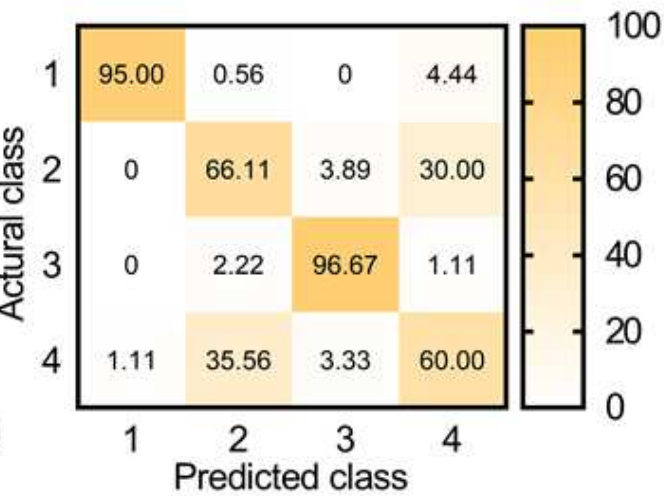

(f)

Figure 20

Fault diagnosis results of two feature extraction sets using the HHO-SVM: (a) MGRCMSE fitness curve, (b) MGRCMSE output results, (c) MGRCMSE confusion matrix (\%), (d) MGMSE fitness curve, (e) MGMSE output results, and (f) MGMSE confusion matrix (\%) 


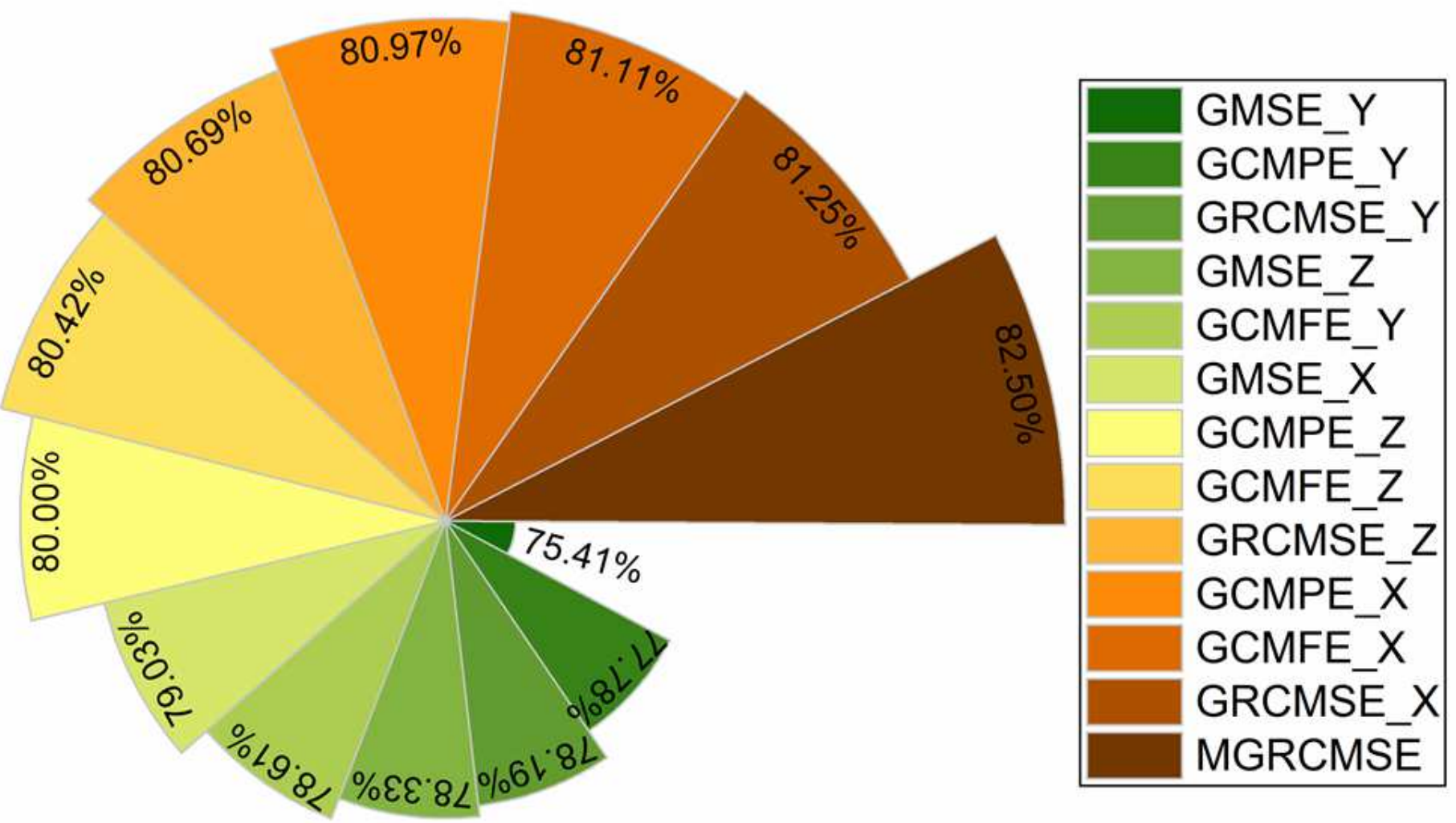

Figure 21

Fault diagnosis accuracy of various feature extraction algorithms 